Pay Transparency under Subjective Performance Evaluation

by

Matthias FAHN

Giorgio ZANARONE

Working Paper No. 2102

January 2021

Johannes Kepler University of Linz Department of Economics Altenberger Strasse 69 A-4040 Linz - Auhof, Austria www.econ.jku.at 


\title{
Pay Transparency under Subjective Performance Evaluation
}

\author{
Matthias Fahn and Giorgio Zanarone*
}

January 2021

\begin{abstract}
This paper studies how pay transparency affects organizations that reward employees based on their efforts (i.e., using "subjective performance evaluation"). First, we show that transparency triggers social comparisons that require the organization to pay its employees an "envy premium". This premium reduces the value of the employment relationship to the organization, and thus its incentive to pay subjective bonuses to the hard-working employees. To restore credibility of its incentive system, a transparent organization must therefore reduce the weight of bonuses, and increase the weight of fixed salaries, in the employees' compensation, relative to organizations that operate in a more conventional "pay secrecy" regime. Second, we show that transparency enables the employees to collectively sanction the organization for reneging on subjective incentives. Collective enforcement allows the transparent organization to use strong employment relationships to "cross-subsidize" weak ones, achieving a more balanced allocation of effort than under pay secrecy. We discuss testable implications of our model for compensation design, the choice between transparency and secrecy regimes, and organizational responses to pay transparency laws.
\end{abstract}

Keywords: Social Comparisons, Secrecy, Transparency, Relational Contracts, Incentives.

JEL Classification: D03, D23, M52, M54.

\footnotetext{
* Matthias Fahn, Johannes Kepler University Linz, e-mail: matthias.fahn@ @ jku.at; Giorgio Zanarone, Washington University in St. Louis, e-mail: gzanarone@ wustl.edu. We thank Nicholas Argyres, Bob Gibbons, Leshui He, Birger Wernerfelt, and participants to the MIT organizational economics lunch, for useful comments. This study received financial support from the Spanish Ministry of Economy and Competitiveness through grant ECO2017-85763-R.
} 


\section{Introduction}

Do organizations benefit from informing employees on their peers' pay? How does pay transparency affect employees' incentives and performance? These are important, yet controversial questions. While prominent consultants and managers - most recently, the founder and CEO of Netflix - have advocated the motivational benefits of transparency (Hastings and Meyer, 2020), employers are reportedly reluctant to embrace this policy (Gely and Bierman, 2003; Edwards, 2005; Hill, 2016).

Scholarly research does not provide clear guidance to organizations on this matter (Colella et al., 2007). The empirical evidence is rather mixed, suggesting that transparency has both positive and negative effects on incentives. Some studies indicate that secrecy weakens employees' incentives because it reduces their trust in the employer and their ability to estimate the link between pay and performance (Futrell, 1978; Lawler, 2000; Bamberger and Belongowski, 2010; Belogolowski and Bamberger, 2014). Other studies, however, show that transparency reduces the satisfaction (Card et al., 2012) and effort (Cohn et al., 2014) of employees with lower pay, possibly because it fosters envious social comparisons between them (Nickerson and Zenger, 2008). Relatedly, recent evidence shows that transparency discourages the use of output-based incentives (Ockenfels et al., 2015; Gartenberg and Wulf, 2017; Obloj and Zenger, 2020). Yet, despite these conflicting empirical findings, there is surprisingly little theoretical research analyzing the benefits and costs of pay transparency for incentive provision in organizations.

Our paper contributes to fill this gap between empirical and theoretical research on pay transparency. It does so by developing a game-theoretic model of the determinants and consequences of transparency in firms that conduct subjective performance evaluations (hereafter, SPE) of their employees. Our model features one principal and two identical agents, each of whom exerts productive effort that benefits the principal. We model SPE as a self-enforcing (hereafter, "relational") contract in which the principal - but not outsiders, such as courts - observes each agent's effort, after which it chooses whether to retain the agent and pay him a bonus. Agents are therefore motivated to exert effort by a future "stick" (the threat of 
being terminated) and an immediate carrot (the bonus). As a result, SPE suffers from a well-known credibility problem: after observing high efforts, the principal has no incentive to pay bonuses unless its profit from continuing the employment relationship with the agents is high enough (Bull, 1987; MacLeod and Malcomson, 1989; Levin, 2003).

SPE is an important context for studying the effect of transparency on incentives, for two reasons. First, piece rates and output-based incentives are often dysfunctional and prone to gaming (Kerr, 1975; Holmstrom and Milgrom, 1991; Larkin, 2014). As a result, SPE plays a central role in the incentive systems of modern organizations, especially for managerial and professional jobs (e.g., Hayes and Schaefer, 2004; Gibbs et al., 2004; Gillan et al., 2009; Frederiksen et al., 2017). Second, according to the literature on personnel psychology and human resources, a major benefit of pay transparency is that it strengthens employees' trust in the organization's subjective performance assessments (e.g., Lawler, 2000; Belogolowski and Bamberger, 2014). Investigating the robustness and boundaries of this proposition is therefore an important goal for a theoretical analysis of transparency.

The key contribution of our model is to show that pay transparency generates both costs and benefits for incentive provision via SPE. On the cost side, we follow an extensive literature in management and economics and assume transparency uncovers pay differences among the two similar agents, which stimulates envious social comparisons between them (e.g., Akerlof and Yellen, 1990; Fehr and Schmidt, 1999; Nickerson and Zenger, 2008; Gino and Pierce, 2009, 2010; Card et al. 2012; Edelman and Larkin, 2014; Obloj and Zenger, 2017). Unlike in objective performance evaluation systems - where pay differences and social comparisons are triggered by ex post differences in output realizations - we argue that in a SPE system, pay differences among otherwise similar agents are triggered by ex-ante differences in the agents' alternative employment opportunities (e.g., due to mobility). We show that the social comparisons stimulated by transparency undermine SPE because they force the principal to pay an "envy premium" to the agents, which reduces the value of the employment relationships to the principal, and hence her incentive to reward high efforts via bonuses. Next to this potential cost of transparency, we model 
a benefit that is rarely remarked, yet crucial for SPE: organizational accountability. Specifically, we argue that by making bonus payments visible to both agents, transparency enables multilateral - as opposed to bilateral - enforcement of their relational contract with the firm (Levin, 2002): if the principal fails to reward a hard-working agent with a bonus, the other agent will lose trust in the organization and quit. ${ }^{1}$

Analyzing the interplay between accountability benefits and social comparison costs of transparency, our model generates three sets of results. The first one pertains to the effects of transparency on effort and output. We find that under pay secrecy, the principal elicits more effort from the agent with lower outside option because this agent receives a lower pay than his peer, and hence the principal stands to gain more from paying the promised bonus and continuing the relationship with him. In contrast, under transparency, the principal can elicit the same effort from the two agent and as a result, produces more output. This efficient reallocation of effort is due to multilateral enforcement: a transparent firm can use the more valuable relationship with the low-outside-option agent to "cross-subsidize" the less valuable relationship with the high-outside-option agent, thereby enhancing the credibility of SPE and its own ability to allocate effort efficiently.

Our second set of results pertains to how transparency affects pay structure under SPE. Compared to pay secrecy, we show that under transparency the principal relies more on the fixed salary, and less on the bonus, to incentivize the envious agent. The reason is that under transparency, a high salary provides that agent with a double incentive to exert effort - first, the agent is afraid of being terminated and losing his salary in the future (the "stick"), and second, he is less envious of his peer and therefore stands to gain more from cooperation today (the "carrot"). By raising the fixed salary, which she is contractually obliged to pay, the principal can therefore rely less on a discretionary bonus to incentivize the envious agent, and hence soften the credibility problem that plagues SPE. More broadly, our result suggests that transparency and compensation structure (the relative weight of salary and bonus) are complementary tools in a SPE system:

\footnotetext{
${ }^{1}$ Models of multilateral enforcement outside the employment context are Greif et al. (1994), and Spagnolo (1999).
} 
a pay structure that is optimal under secrecy would hamper employee incentives under transparency, and vice versa.

Our third set of results pertains to governance choice. We show that relative to pay secrecy, transparency (combined with a well-adapted pay structure) enhances the overall credibility of SPE, thereby inducing the agents to produce more output, at the cost of increasing the principal's personnel costs due to the need to control social comparisons. Thus, transparency is optimal for the firm when the agents' effort is non-verifiable, such that credibility of SPE is an important concern, when bilateral employment relationships between the principal and the two agents are not strong enough to sustain SPE, and when social comparisons are strong - for instance, due to geographical and social proximity between the agents (Obloj and Zenger, 2017; Gartenberg and Wulf, 2017). While these predictions on the optimal choice between transparency and secrecy await thorough empirical verification, we provide preliminary evidence consistent with one of them. Matching a PayScale survey on transparency in US firms, and Chamber of Commerce data on the quality of judicial enforcement across US states, we find that companies in states with less reliable courts, which are more likely to face credibility problems when promising SPE bonuses to their employees, have higher transparency scores than their peers in high-court-quality states.

The rest of this paper is organized as follows. Section 2 discusses our contributions to the literature. Section 3 presents our baseline model and discusses its assumptions. Section 4 analyzes the optimal design of a SPE system under pay secrecy (section 4.1) and transparency (section 4.2), and the conditions under which transparency is the optimal policy to sustain SPE (section 4.3). Section 5 extends the baseline model - allowing agents to be both "envious" and "compassionate", and limiting the principal's ability to make performance assessments, in addition to pay, transparent - and shows robustness of the key results. Section 6 concludes. 


\section{Contributions to the literature}

\subsection{Models of pay transparency vs. secrecy}

As discussed in the introduction, ours is one of the first formal models comparing pay secrecy to transparency. A related paper is Cullen and Pakzad-Hurson (2020), which models the effect of pay transparency on wage setting in the presence of asymmetric information. Their paper is complementary to ours, in that it focuses on different determinants and consequences of transparency. While we study how transparency affects incentives, Cullen and Pakzad-Hurson (2020) abstract from incentive issues, and focus instead on how transparency alters the relative bargaining power of workers and firms. Moreover, unlike us, Cullen and Pakzad-Hurson (2020) do not analyze the social comparison costs of transparency. Lastly, while our model naturally applies to employees incentivized through SPE, such as managers, both the theoretical and empirical analysis of Cullen and Pakzad-Hurson (2020) are tailored to temporary workers.

\subsection{Social comparisons in organizations}

Our paper also relates to an emerging literature that analyzes organizational responses to social comparisons. Most studies in management focus on the relationship between social comparisons and compensation design. For instance, Larkin et al. (2012) argue theoretically that social comparisons raise the organizational costs of pay-for-performance, and that firms may resort to alternative compensation schemes, such as seniority-based and flat wages, to mitigate these costs. Consistent with these predictions, several empirical studies have found a positive association between social comparisons, wage compression, and a reduced use of pay-for-performance (Wade et al., 2006; Shue, 2013; Gartenberg and Wulf, 2017; Obloj and Zenger, 2017; Mas, 2017). A few studies have looked at the complementary issue of how social comparisons affect firm boundaries. Nickerson and Zenger (2008) argue that social comparisons are more pronounced within firms than between and therefore represent a central cost of firm scope. Consistent with 
their theory, recent studies have found that intra-firm social comparisons lead to divestitures (Feldman et al., 2018) and to greater pay compression within firms than between (Gartenberg and Wulf, 2019).

In economics, the earliest theoretical analyses of social comparisons were offered by Fehr and Schmidt (1999), and Bolton and Ockenfels (2000). Englmaier and Wambach (2010) first incorporated social comparisons into a model of incentive contracts. Grund and Sliwka (2005) analyze the roles of upwards social comparisons (labeled as “envy”) and downwards comparisons (labeled as “compassion”) in incentive tournaments. Closer to us, Kragl and Schmid (2009), discussed in greater detail below, analyze how envy affects output-based relational incentives. All of these papers assume pay transparency so unlike our model, they do not compare pay structure and firm performance under transparency vs. secrecy. Additionally, these papers differ from our model in that they do not study the effect of social comparisons on SPE.

\subsection{Relational contracts}

Lastly, our paper contributes to the literature on relational contracts (in the sense of self-enforcing agreements sustained by repeated interactions). A stream of this literature studies how an organization's formal governance structure (including contracts with employees and independent partners) affects its ability to sustain self-enforcing agreements (Klein, 2000; Poppo and Zenger, 2002). Theoretical contributions to this literature have analyzed how relational contracts interact, among others, with formal incentive pay (Baker et al., 1994), firm boundaries (Baker et al., 2002), the allocation of control (Baker et al., 2011; Zanarone, 2013), and the scope of partnerships (Argyres et al., 2020). The complementarity between formal governance and relational contracts has also been supported by empirical studies on interfirm collaborations (e.g., Poppo and Zenger, 2002; Ryall and Sampson, 2009; Kosova and Sertsios, 2018; Barron, Gibbons, Gil and Murphy, 2019; Gil, Kim and Zanarone, 2021). ${ }^{2}$ While existing theories focus on how formal allocations of control and income rights (via asset ownership or contracts) affect relational

\footnotetext{
${ }^{2}$ See Cao and Lumineau (2015), and Gil and Zanarone $(2017,2018)$, for complementary reviews of the evidence.
} 
agreements, our paper focuses on a novel aspect of formal governance - informational restrictions on pay - and shows that it importantly affects relational collaboration.

Additionally, our paper is among the first to incorporate social preferences into models of relational incentive contracts. Dur and Tichem (2015) demonstrate that altruistic preferences of supervisors (towards their subordinates) may harm organizations by eroding the credibility of termination threats as a means to provide incentives. Fahn (2020) analyzes a relational contracting model with reciprocal employees, and shows that it is optimal for the firm to pay generous fixed wages to employees who are close to retirement, and performance-contingent bonuses to those in earlier stages of their career. Fahn et al. (2017) provide empirical evidence consistent with this prediction.

Perhaps the closest to our model is Kragl and Schmid (2009), which analyzes relational incentive contracts in the presence of envy (that is, implicitly assuming a regime of pay transparency). Unlike in our paper, in Kragl and Schmid (2009) agents are ex ante identical and receive output-based, rather than subjective bonuses (that is, there is no SPE in their paper). As a result, social comparisons only arise there if due to stochastic output realizations, one agent receives a bonus while the other does not. The key insight is that agents can decrease their chances of suffering from social comparisons by working hard, and as a result, envy strengthens their incentives, relaxes the firm's credibility problem, and can thus potentially increase profits. Kragl and Schmid (2009) differs from our paper in three important respect. First, given its focus on ex ante identical agents and output-based incentives, their model does not analyze social comparison costs under SPE. Second, they do not explore the effect of social comparisons on pay structure. Lastly, as discussed above Kragl and Schmid (2009) do not study the differential effect of pay transparency and secrecy on relational incentives. 


\section{Model}

\subsection{The organization}

Consider an organization that consists of three individuals: a principal (she), agent 1 (he), and agent 2 (he), all risk-neutral. Time is discrete, the time horizon is infinite, and the principal and both agents discount next-period payoffs by a common factor $\delta \in(0,1)$. Before the relationship with the two agents starts, the principal permanently commits to a policy $s \in\{0,1\}$, where $s=1$ denotes "secrecy", and $s=0$ denotes "transparency". Both policies will be precisely defined in a moment. Once the policy is chosen, the principal and the agents interact in each period $t=1,2, \ldots$ as described by Figure 1 below.

\section{Figure 1. Timeline}

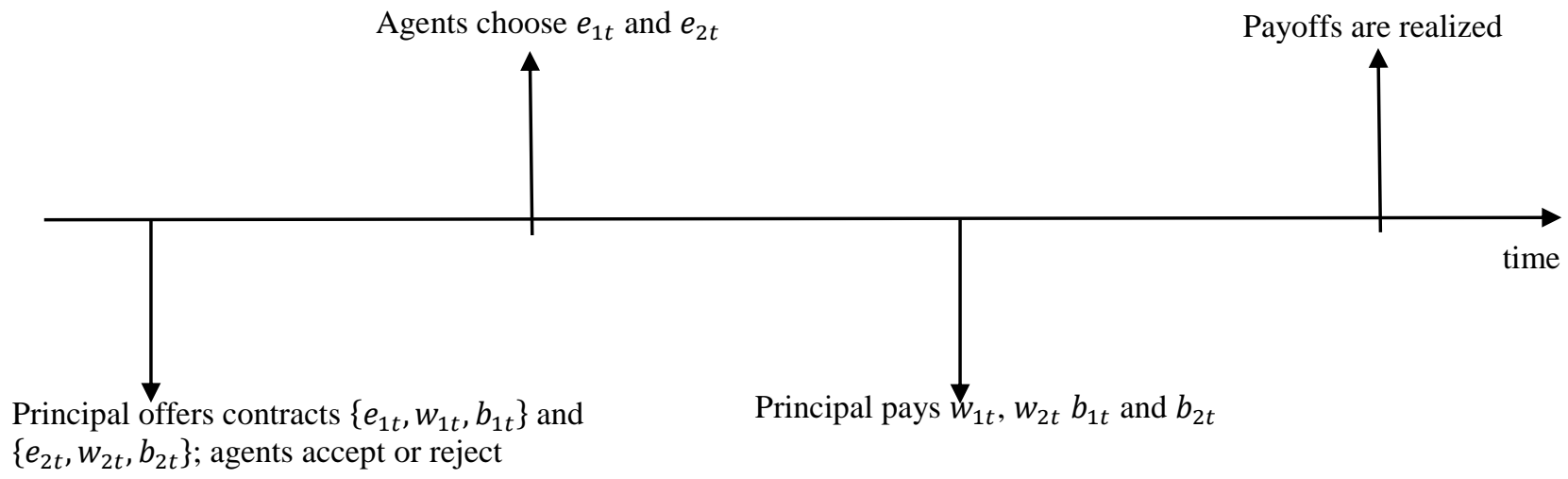

At the beginning of period $t$ the principal offers an employment contract $\left\{e_{i t}, w_{i t}, b_{i t}\right\}$ to each agent $i \in\{1,2\}$, where $e_{i t} \geq 0$ is the agent's required labor input (hereafter, "effort"), $w_{i t} \in \mathbb{R}$ is a formal (i.e., court-enforceable) fixed salary, and $b_{i t} \geq 0$ is an informal (i.e., discretionary) bonus contingent on effort. Given our focus on subjective performance evaluation, we therefore model incentive provision as "pay-forinput," as in the case of executives who receive a discretionary bonus contingent on performance assessment by the board (Hayes and Schaefer, 2004), or employees who are individually evaluated by their 
supervisors based on fine-tuned performance indicators (Gibbs et al., 2004; Kampkotter and Sliwka, 2015 Frederiksen et al., 2017).

If agent $i$ accepts the proposed contract, he chooses whether to exert the agreed upon effort $e_{i t}$ at cost $c\left(e_{i t}\right)$. After observing effort, the principal pays the formal salary $w_{i t}$ and chooses whether to pay the agreed upon bonus, $b_{i t}$. Finally, at the end of the period the principal receives the output generated by the two agents through their efforts, $y\left(e_{1}, e_{2}\right)=y\left(e_{1}\right)+y\left(e_{2}\right)$. We assume that for each agent $i \in\{1,2\}$, the effort cost is strictly increasing and convex $\left(c^{\prime}\left(e_{i}\right)>0, c^{\prime \prime}\left(e_{i}\right)>0\right.$, with $\left.c(0)=c^{\prime}(0)=0\right)$, and output is strictly increasing $\left(y_{i}>0\right)$, weakly concave $\left(y_{i i} \leq 0\right)$, and satisfies $y(0)=0$.

If agent $i$ rejects the principal's offer, he receives an outside option $\underline{u}_{i}$, and the principal receives $\underline{\pi}$. Importantly for our subsequent analysis of social comparisons, we assume the agents have different outside options: $\underline{u}_{1}>\underline{u}_{2}$. Without loss of generality, we normalize $\underline{\pi}$ and $\underline{u}_{2}$ to zero, such that $\underline{u}_{1}$ can be interpreted as the outside option differential between the two agents. For instance, agent 2 may face higher relocation costs because of his personal situation (married, with children, etc.), or may be less productive than agent 1 in alternative jobs (as an example close to home, think of the different non-academic options of an applied microeconomics professor as opposed to a corporate finance professor).

We conclude the model's setup by stating our informational assumptions and by providing a precise definition of transparency and secrecy and of how they affect the agents' information. First, we assume efforts are observed by the principal but may not be verifiable by third parties outside the organization, such as courts. We briefly analyze below the case of verifiable efforts, in which the principal can make formal salaries contingent on the observed effort levels, and then we turn to the more interesting case of nonverifiable efforts in which the principal can make the informal bonuses, but not the formal salaries, contingent on effort. We will show that effort verifiability importantly affects the choice between pay transparency and secrecy.

Second, we assume in our baseline model that an agent's information on his peer's contract, effort and received compensation depends on the policy chosen by the principal, as follows. Under transparency $(s=$ 
0 ), each agent $i \in\{1,2\}$ in each period $t$ observes: (1) the contract offered to the other agent $j$, $\left\{e_{j t}, w_{j t}, b_{j t}\right\},(2)$ whether agent $j$ has exerted the agreed upon effort, and (3) whether the principal has paid the agreed upon compensation $w_{j t}+b_{j t}$ to agent $j$. Conversely, under secrecy $(s=1)$, agent $i$ observes nothing about the contract, effort and received compensation of agent $j .{ }^{3}$ We therefore interpret transparency as a policy in which the principal discloses information about contractual conditions and pay in the organization ("vertical" transparency) and simultaneously promotes social interactions and communication among the agents, such that these end up observing both how much their peer is paid and how much he works ("horizontal” transparency). ${ }^{4}$ We interpret secrecy in a specular way.

Our assumption that the principal can make effort observable to peers by design is consistent with the practice of highly transparent organizations, such as Netflix (Hastings and Meyer, 2020). Yet, Netflix-style organizational practices may be costly and not accessible or well suited to other firms. In section 4.1, we therefore analyze the case in which the agents' ability to observe their peer's effort is exogenously given, and the principal's choice between secrecy and transparency solely determines whether agents can observe their peers' contract and compensation.

\subsection{Payoffs and social comparisons}

Given the definitions above, the principal's profit in period $t$ when both agents accept to work is:

$\pi_{t} \equiv y\left(e_{1 t}, e_{2 t}\right)-w_{1 t}-w_{2 t}-b_{1 t}-b_{2 t}$

Social comparisons make definition of the agents' utilities more complex. On the one hand, as in standard models of employment, each agent cares about his "material payoff", that is, total compensation minus the cost of effort:

$$
m_{i t} \equiv w_{i t}+b_{i t}-c\left(e_{i t}\right), \text { for } i \in\{1,2\}
$$

\footnotetext{
${ }^{3}$ Contreras, Fahn and Zanarone (2019) analyze a model in which pay information may occasionally leak under secrecy. They study how a firm should design compensation to implement pay secrecy under these circumstances. ${ }^{4}$ This "holistic" view of transparency is consistent with the idea, emphasized in the literature (e.g., Gely and Bierman, 2003; Edwards, 2005; Cullen and Perez-Truglia, 2019), that pay disclosure by the principal (the lack thereof) is ineffective in the absence of a broader organizational culture that consistently promotes (discourages) communication and transparency among the employees.
} 
On the other hand, unlike in standard models, we assume that agents engage in "envious" social comparisons, in the sense that they suffer when their material payoff is lower than that of the other agent, (e.g., Nickerson and Zenger, 2008; Larkin et al., 2012). To formally capture this idea we adapt established models of social preferences (e.g., Fehr and Schmidt, 1999; Grund and Sliwka, 2005; Englmaier and Wambach, 2010) and assume that when payoff differences are observed (that is, under transparency), an individual agent's utility decreases by:

$$
\eta \max \left\{0, m_{j t}-m_{i t}\right\} \text {, for agent } i \in\{1,2\}
$$

Thus, social comparisons imply that an agent's utility from working in the organization crucially depends on the principal's choice between transparency and secrecy:

$$
u_{i t} \equiv m_{i t}-(1-s) \eta \max \left\{0, m_{j t}-m_{i t}\right\} \text {, for } i \in\{1,2\} \text {. }
$$

Note that we model envious agents because a strong and growing body of empirical evidence suggests individuals in organizations suffer from unfavorable social comparisons but do not care much about favorable ones (e.g., Card et al., 2012; Cohn et al., 2014; Ockenfels et al., 2015; Breza et al., 2018). Nevertheless, one may argue that in some settings agents might be symmetrically averse to inequality - that is, they might suffer from pay differences even when these favor them (e.g., Grund and Sliwka, 2005; Englmaier and Wambach, 2010; Ashraf, 2018). ${ }^{5}$ In section 4.2 we therefore extend the model to this case and show that our main insights continue to apply.

\subsection{Discussion of the assumptions}

Some features of our model deserve further discussion. First, we have assumed that social comparisons are triggered by observed payoff differences rather than by employees' inferences about equilibrium differences - that is, employees "believe what they see". This assumption allows us to analytically capture the intuitive idea that pay secrecy may reduce social comparisons. We are comfortable with this assumption because it is supported by psychological research on self-serving beliefs (e.g., Kunda, 1990), which

\footnotetext{
${ }^{5}$ See Ashraf and Bandiera (2018) for a literature review that discusses both favorable and unfavorable comparisons.
} 
suggests that individuals construct beliefs that make them better off so long as these are not inconsistent with the available evidence. In our model this notion implies that absent information on payoff differences between the agents (i.e., under secrecy), the agents will convince themselves to be equal, thus avoiding any disutility from social comparisons.

Second, we have assumed the two agents are equally productive and that their individual contributions to output can be separated. Equal productivity simplifies the analysis but does not affect the model's results. Moreover, this assumption seems natural in a model in which transparency generates social comparison costs. If besides having a higher outside option agent 1 were also more productive than his peer, then agent 2 might be more willing to accept a pay differential without suffering from envy (Breza et al., 2018).

Output separability does not matter for our results on the optimal pay structure and the comparative statics of agent efforts under pay transparency, analyzed in section 4.2 (to appreciate that, one only needs to check that the separability assumption is not used anywhere in the proofs of propositions 3 and 4). The fact that results on transparency continue to hold under team production is reassuring because transparency (on both pay and mutual performance, as in our baseline model) is arguably easier to implement in settings where employees closely interact.

The separability assumption does matter, however, for our analysis of secrecy (and hence for its comparison to transparency in section 4.3). If the two agents' efforts were complementary inputs $\left(y_{12}>\right.$ 0 ), termination of the relational contract with one agent would reduce the principal's future payoffs from her relationship with the other agent. This, in turn, would strengthen the punishment against principal's breach under secrecy, reducing the need for transparency as a means to make the principal more accountable. We assume separability because we find it conceptually difficult to envision subjective performance evaluation under pay secrecy in the presence of strong team complementarities, for two reasons. First, team production is normally associated with interaction and proximity between employees, which undermines the organization's ability to keep pay secret. Second, the fact that the values of the two employment relationships are interdependent under team production would force each agent to think of his peer's contract terms when assessing the principal's "trustworthiness" (i.e., her incentive to renege on 
discretionary pay). ${ }^{6}$ That, however, would defeat the purpose of secrecy - namely, to prevent social comparisons between employees. We therefore believe that when the principal uses SPE to motivate agents, and hence it is important for the agents to assess the principal's willingness to pay the promised incentives, a conceptually coherent theory of pay secrecy requires that the agents separately contribute to the organization, such that their relationships with the principal under secrecy are truly bilateral.

\section{Subjective incentives under secrecy and transparency}

In this section we use our model to analyze SPE under both secrecy and transparency and the conditions under which one or the other policy is optimal. As a benchmark we begin by studying the simple case in which the agents' efforts are verifiable and derive a contract that maximizes the principal's profit. In that case, the principal can induce agents to exert the desired effort levels in every period by conditioning their formal salaries to those efforts. Bonuses are not necessary to incentivize the agents and can thus be ignored.

Let $e_{1}^{F B}=e_{2}^{F B}=e^{F B}$ be the efficient or "first best" efforts that maximize net total surplus in the absence of social comparisons, $y\left(e_{1 t}, e_{2 t}\right)-c\left(e_{1}\right)-c\left(e_{2}\right)$. We then have the following result.

Proposition 1. With verifiable efforts, secrecy achieves the first best and is therefore optimal.

\section{Proof: In Appendix A.}

This is an intuitive result. Under secrecy the agents do not observe any information on their peer's material payoff and therefore do not suffer from social comparisons. Thus, the principal can induce them to exert first best efforts, and appropriate the resulting surplus, by agreeing to pay each agent a formal salary

\footnotetext{
${ }^{6}$ The subtleties of this argument will be clearer once the model has been fully analyzed. As shown below, under secrecy the principal has an incentive to pay the promised bonus to an agent if her profit loss from terminating the relationship with him, while continuing that with the other agent, is large enough. With separate output contributions this profit loss is simply equal to the focal agent' $\mathrm{s}$ individual output minus his total pay, so in order to assess the principal's trustworthiness, the agent only needs to know his own terms of employment (salary, bonus and required effort). In contrast, with effort complementarities the agent also needs to think about how his peer's terms of employment would change if the principal reneged because by causing the focal agent to quit, the principal's breach would reduce the team's size and hence the peer's productivity. As he tries to anticipate changes in his peer's terms of employment however, the agent would likely suffer from social comparisons and the rationale for having secrecy in the first place would disappear.
} 
that covers his outside option plus effort cost, $w_{1}=\underline{u}_{1}+c\left(e^{F B}\right)$ and $w_{2}=c\left(e^{F B}\right)$, if the agent exerts effort $e^{F B}$, and zero if the agent shirks. Transparency, on the other hand, delivers no additional benefits but triggers social comparison costs due to the fact that the agents have different outside options and hence the principal would like to pay them different salaries for a given level of effort. Thus, transparency cannot be optimal.

We now turn to the more interesting case in which the agents' efforts are observed by the principal (and may be assessed by peers under transparency) but cannot be verified by parties outside the organization, such as courts. To implement SPE, the principal must now rely on "relational" incentive contracts in which she promises to pay each agent a bonus following the desired effort level, and the agent trusts the principal to honor this promise.

Following an extensive literature (e.g., MacLeod and Malcomson, 1989; Levin 2002, 2003), we model relational contracts as subgame perfect equilibria of the repeated game in which deviations (i.e., low effort by an agent or non-payment of a bonus by the principal) are "punished" optimally - that is, by termination of the relationship in subsequent periods, which in our context is the harshest credible punishment (Abreu, 1988). ${ }^{7}$ We focus on relational contracts that are "stationary" (i.e., prescribe the same efforts and payments in every period), and "optimal" (i.e., yield the maximum payoff to the principal among all stationary contracts). We hereafter drop all time subscripts accordingly. The stationarity assumption is without any loss of generality in our model of secrecy (following arguments delivered by Levin, 2003), and it is without loss for all periods of the employment relationship but the first one in the model of transparency. Since we are mainly interested in the properties of ongoing employment relationships, we are comfortable with making the stationarity assumption throughout.

Our key insight is that the principal's policy (transparency vs. secrecy) determines how harshly the agents can punish the principal for reneging on a relational contract, and therefore affects the effort levels

\footnotetext{
${ }^{7}$ Some relational contracting models assume that parties revert to the optimal spot (formal) contract forever following a deviation (e.g., Baker et al., 1994, 2002). In our context the two approaches are equivalent because termination is the optimal spot contract: absent relational contracting the agents spend zero effort and generate zero surplus in every period so the principal is better off not hiring them.
} 
that can be sustained in equilibrium under SPE. Under secrecy, whether the principal has failed to pay the promised bonus after the agent has exerted the promised effort can only be observed and punished (via termination) by the affected agent - that is, relational contracts are bilateral (as in Levin, 2003). In contrast, under transparency, the principal's breach can be observed and punished by both agents - that is, relational contracts are multilateral (as in Levin, 2002). This rarely remarked benefit of transparency - facilitating collective action by turning relational employment contracts from bilateral into multilateral counterbalances social comparison costs, thereby creating a tradeoff between the two policies.

Notice that multilateral enforcement under transparency is not only optimal from a game-theoretic viewpoint, but also consistent with practice. Psychologically, we see multilateral enforcement as a natural consequence of the agents' loss of trust in the organization. Each agent believes that if the principal has failed to pay the promised bonus to a colleague, she may do the same to him in the near future. Thus, the organization's principles "sold" to agents upon entry (in our case, the principle that efforts are rewarded with bonuses) are no longer credible, and the agents quit and look for a more reliable employer. Indeed, organizations perceived to violate their own principles have often experienced massive retaliation not limited to the employees individually affected by the specific violation. For instance, First Boston lost most of its top managers, including the CEO, and was unable for some time to recruit business school graduates, after it paid lower-than-expected bonuses to its investment bankers in 1992 (Stewart, 1993). Similarly, Oticon (a leading manufacturer of hearing aid equipment) experienced generalized loss of morale and a decline in innovation after the top management rejected numerous new product candidates proposed by internal teams, thereby violating the company's loudly announced culture of delegation and employee empowerment (Foss, 2003).

\subsection{Incentive provision under secrecy}

Under secrecy, there are no social comparisons between agents, and relational employment contracts are bilateral. In such a setting, the principal hires each agent, and motivates him to work, by paying a formal salary equal to the agent's outside option, plus a discretionary incentive bonus equal to his effort cost 
(details are in the proof of Proposition 2). Thus, the optimal relational contract prescribes effort levels that maximize the principal's profit,

$$
\pi \equiv y\left(e_{1}, e_{2}\right)-w_{1}-w_{2}-b_{1}-b_{2}=y\left(e_{1}, e_{2}\right)-c\left(e_{1}\right)-c\left(e_{2}\right)-\underline{u}_{1},
$$

subject to the principal being willing to pay the promised bonuses after efforts are sunk (enforceability constraints):

$$
\begin{aligned}
& \frac{\delta}{1-\delta}\left(y\left(e_{1}\right)-c\left(e_{1}\right)-\underline{u}_{1}\right) \geq c\left(e_{1}\right), \text { and } \\
& \frac{\delta}{1-\delta}\left(y\left(e_{2}\right)-c\left(e_{2}\right)\right) \geq c\left(e_{2}\right) .
\end{aligned}
$$

The left-hand sides of (2) and (3) measure the strength of the principal's employment relationships with the two agents, given by the discounted net profits the principal would lose upon termination. The righthand sides measure the principal's "reneging temptation" - that is, her immediate savings from not paying the promised bonus to each agent. Importantly, for a given effort level, the principal attaches higher value to the relationship with, and hence can elicit more effort from agent 2 because agent 2 has a lower outside option, and hence receives a lower salary, than agent 1 .

Proposition 2. With non-verifiable efforts, optimal contracts under secrecy have the following characteristics. (1) Each agent receives a salary equal to his outside option and a bonus equal to the effort cost. (2) When bilateral employment relationships are strong (high $\delta$ ) both agents exert efficient effort. (3) When employment relationships are weak (low $\delta$ ), agent 1 exerts lower effort than agent 2.

\section{Proof. In Appendix A.}

Given the absence of social comparisons, the only source of inefficiency under secrecy is nonverifiability of the efforts. This forces the principal to motivate agents through subjective bonuses, whose credibility is constrained by the lack of court enforcement. Then, proposition 2 confirms the tenet that an organization can elicit greater effort from an employee via SPE the more it stands to lose from termination of the relationship (e.g., MacLeod and Malcomson, 1989; Levin, 2003). When the value of the relationship is high enough, the employee "trusts" the organization to honor its bonus promise and is therefore motivated 
to perform. Moreover, all else equal, the employee with lower outside option works harder because his relationship with the organization has higher net value.

\subsection{Incentive provision under transparency}

When switch to transparency, implementation of SPE changes in two ways. First, since agent 2 envies his peer, the principal must pay him an "envy premium". Second, if the principal fails to pay the bonus to one agent, her relationship with both agents terminates because the other agent observes the principal's defection. Formally, the two enforceability constraints we observed under secrecy are now merged:

$$
\frac{\delta}{1-\delta} \pi \geq b_{1}+b_{2}
$$

Thus, on the one hand, transparency imposes social comparison costs on the firm. On the other hand, by increasing the firm's accountability, transparency potentially enhances its flexibility in providing incentives via SPE. Our next proposition, and figure 1 below, illustrate this point.

\section{Figure 1: Agents' efforts under transparency}

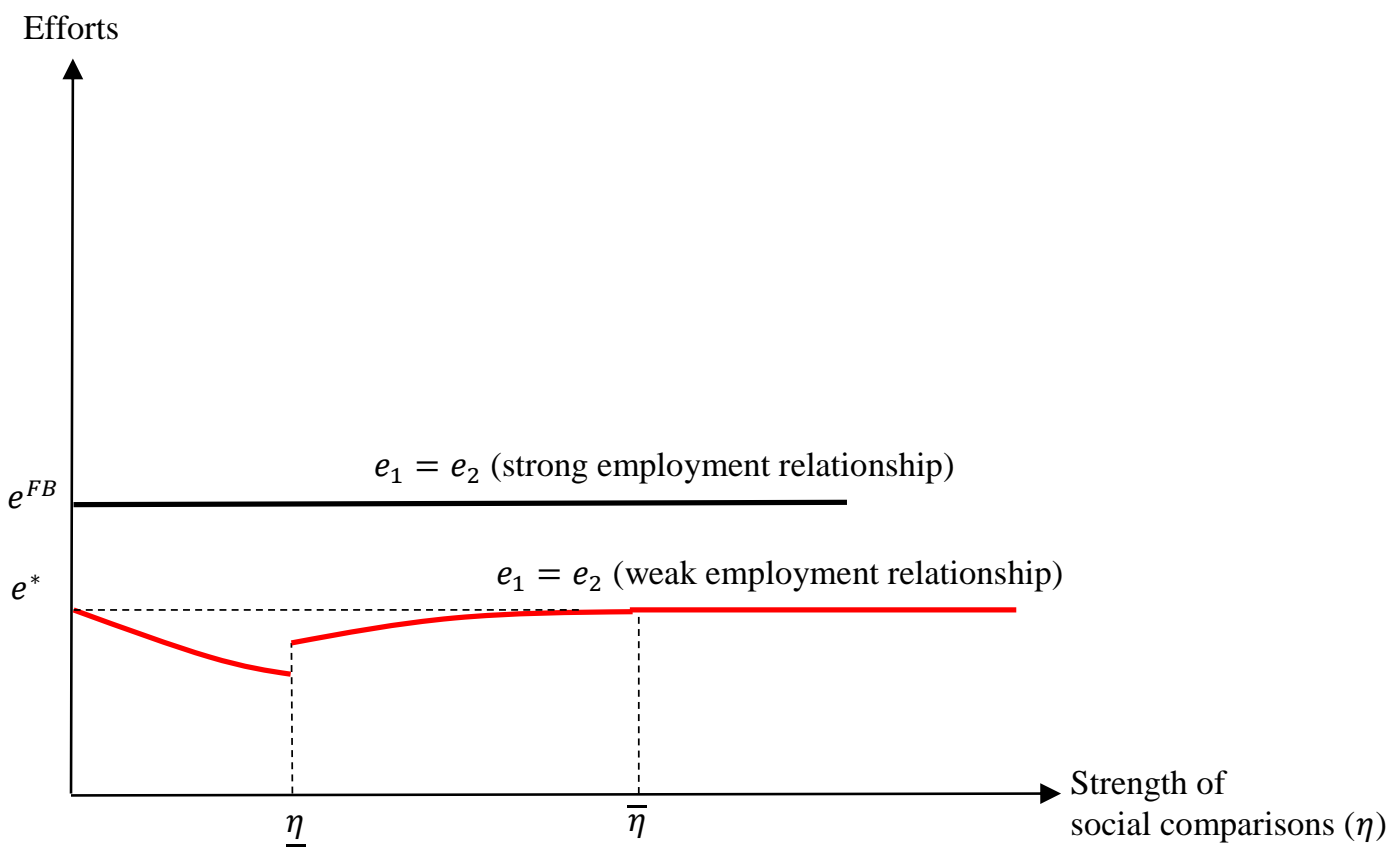


Proposition 3. (1) Under transparency, both agents exert the same effort. (2) If the employment relationship is strong (high enough $\delta$ ), this effort is set at the efficient level. (3) If the employment relationship is weak (low $\delta$ ), the agents' effort is inefficiently low. Then, effort initially decreases in the strength of social comparisons, and becomes (weakly) increasing when social comparisons are strong enough.

\section{Proof: in Appendix A.}

In weak employment relationships, the principal does not have enough credibility to pay bonuses that motivate efficient efforts. In that case, transparency eliminates the effort imbalance we observed under secrecy because it enables multilateral enforcement of relational incentive contracts. Recall that under secrecy, the principal elicits more effort from agent 2 than from agent 1 because agent 2 has a lower outside option, and therefore his bilateral relationship with the principal has a higher net value. This asymmetric allocation of effort is inefficient: given the decreasing marginal productivity of effort, total output would be increased by reducing the effort of agent 2 while increasing that of agent 1 until the two efforts are equal. Under transparency, the principal's obligation to pay bonuses is bounded by the value of both employment relationships, so the principal can rebalance the two efforts by using the stronger relationship with agent 2 to "cross-subsidize" the weaker relationship with agent 1 (Bernheim and Whinston, 1990; Levin, 2002).

To understand why effort initially decreases and then increases in social comparisons, we must study the optimal pay structure under transparency, which is illustrated by figures 2 and 3 below. 
Figure 2: Transparency pay structure in strong employment relationship

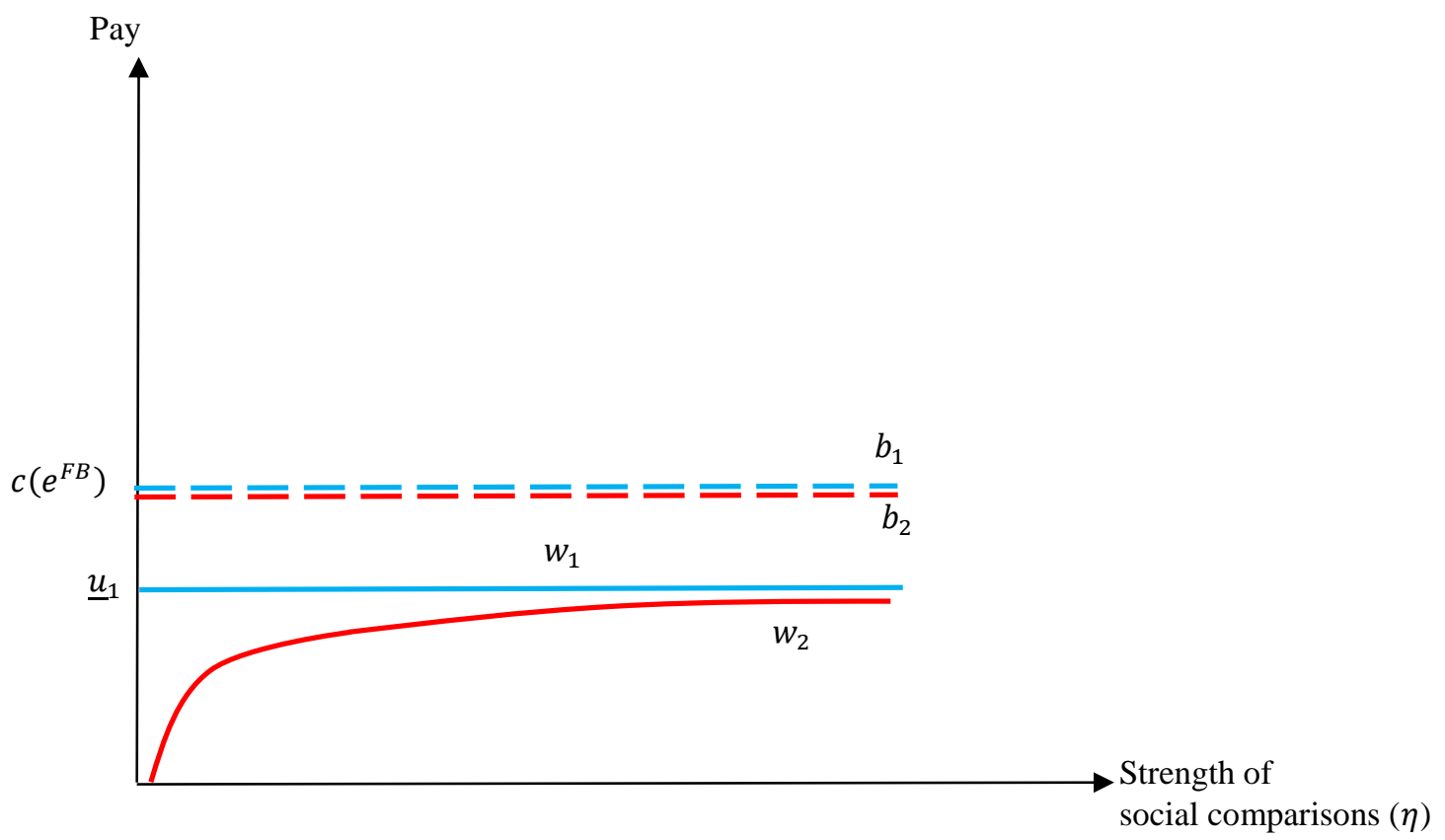

Figure 3: Transparency pay structure in weak employment relationship

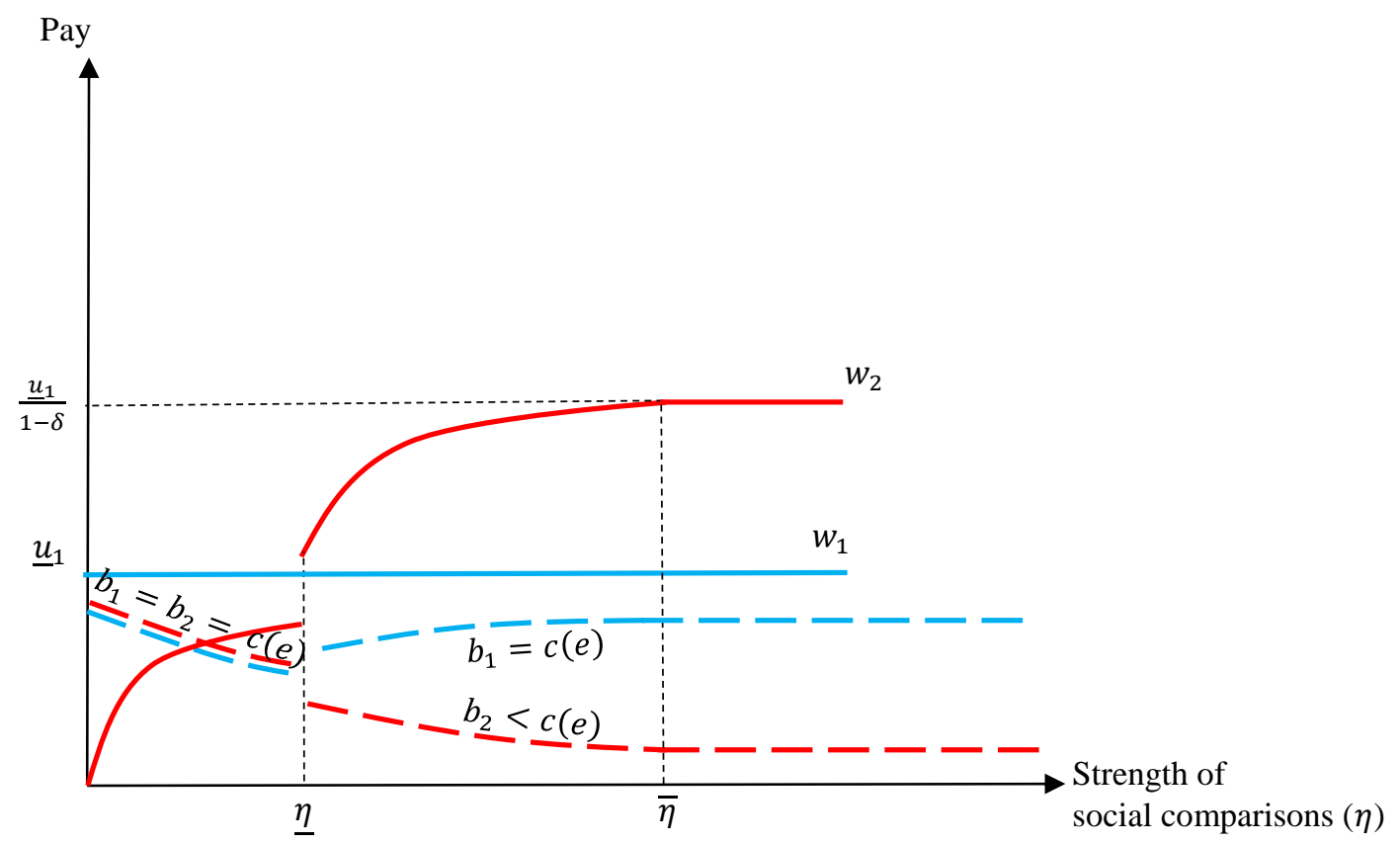


When the employment relationship is strong (high $\delta$ ), the principal motivates both agents to exert efficient efforts through a bonus equal to the effort cost, while paying them the lowest salaries that induce participation. Although neither agent earns a rent under this pay structure (i.e., $u_{1}=\underline{u}_{1}$, and $u_{2}=0$ ), the salary of agent 2 includes an envy premium to compensate for his social comparison disutility: $w_{2}=$ $\frac{\eta \underline{u}_{1}}{(1+\eta)}>0$. This is the pay compression effect analyzed theoretically by Englmaier and Wambach (2010), and verified empirically, among others, by Gartenberg and Wulf (2017), Ockenfels et al. (2017), and Obloj and Zenger (2020).

When the employment relationship is weak (low $\delta$ ), pay structure of the envious agent 2 becomes more complex (whereas that of agent 1 does not change). Now the principal's enforceability constraint is binding, and since the envy premium makes this constraint more stringent, the principal must lower the agents' bonuses, and hence their effort and output, to keep the relational incentive contract self-enforcing. For strong enough social comparisons, this loss of output is so prominent that the principal finds it optimal to give the envious agent 2 a rent $\left(u_{2}>0\right)$ by raising his salary above the outside option differential $\left(w_{2}>\right.$ $\left.\underline{u}_{1}\right)$. By doing so, the principal strengthens the agent's incentives through two mutually reinforcing effects: a higher future payoff from cooperation, which is well known from the efficiency wage literature, and an immediate reduction in the social comparison disutility. This double incentive effect of the fixed salary, in turn, allows the principal to elicit more effort from both agents while reducing the discretionary bonus of agent 2 enough to keep the relational contract self-enforcing. As social comparisons grow larger and larger, the salary of agent 2 eventually becomes high enough to completely eliminate social comparisons. As a result, there is no reason for the principal to further adjust the pay structure.

Our analysis therefore shows that for employees who suffer from strong social comparisons (i.e., agent 2), SPE under transparency puts more weight on fixed salaries, and less weight on discretionary bonuses, compared to secrecy and to standard incentive models that do not feature social comparisons.

Proposition 4. (1) Under transparency, if the employment relationship is strong enough (high $\delta$ ) or if social comparisons are weak enough (low $\eta$ ), agent 2 receives the same bonus as agent 1 , and a lower 
salary. (2) If instead the employment relationship is weak (low $\delta)$ and social comparisons are strong

(high $\eta$ ), agent 2 receives a lower bonus and a higher salary than agent 1 .

\section{Proof. In Appendix A.}

Our results on the optimal pay structure and efforts under transparency importantly diverge from standard models of relational incentive contracts. First, most of these models (e.g., Levin, 2002; Levin, 2003) ignore social comparisons. Consequently, optimal pay structure there puts low weight on fixed salaries, and high weight on discretionary bonuses: the principal incentivizes effort via bonuses, while keeping formal salaries low to increase her own incentive to pay those bonuses. ${ }^{8}$ Second, the very few models of relational incentives that do consider social comparisons (Kragl and Schmid, 2009) do not analyze SPE: there, performance is assessed by the firm based on output rather than observed effort. In those models, social comparisons originate from pay differences due to stochastic output realizations rather than from ex ante differences between agents, as in this paper. Consequently, incentive provision via fixed salaries is not optimal there. ${ }^{9}$

Lastly, while equally productive and hard-working employees may receive different bonuses in standard models (due to different realizations of stochastic output), they should face the same ex ante pay structure. In contrast, in our model employees with the same productivity, and from whom the firm requires the same effort, face different optimal pay structures: the contract of employees with lower outside options gives more weight to fixed pay, and less weight to bonuses, than the contract of employees with higher outside options.

Our predictions also differ from existing management studies of social comparison costs and envy in organizations. These studies (e.g., Nickerson and Zenger, 2008) theorize an unambiguously negative effect

\footnotetext{
${ }^{8}$ In standard relational contracting models, paying a bonus today or an efficiency wage (multiplied by $1 / \delta$ to account for discounting) tomorrow provides the same incentives to both the principal and the agent. Since these models generally focus on stationary contracts, the profit-maximizing mechanism there only uses a bonus, and not a high efficiency wage (which would also have to be paid in the first period of the relationship) to provide incentives. ${ }^{9}$ It would be straightforward to add output-based incentives to our model. Doing so would mitigate the negative effect of social comparisons under transparency, because agents would have an incentive to increase effort in order to minimize expected differences in performance pay. Our current results on pay structure, which stem from the fact that the two agents have different outside options, would continue to hold.
} 
of social comparisons on employee motivation and effort. Our model shows that when subjective performance evaluation is used, severe social comparisons may affect optimal pay structure in a way that increases employees' efforts and output (although social comparisons still reduce firm profits because of upwards pay compression).

Altogether, our model provides novel insights on compensation design in organizations where (1) pay is transparent (due to endogenous internal policy, but also possibly to exogenous constraints such as "sunshine laws" or social norms), and (2) both subjective performance evaluation and social comparisons are important. Testing our predictions (as any predictions involving subjective incentives) is challenging but potentially feasible. First, internal records and survey responses provided by organizations interested in assessing their compensation policies could be used to measure the relative weights of formal and informal pay for different employees (Gibbs et al., 2004). These data could be matched to measures of the strength, expected duration or value of an organization's employment relationships (Gibbs et al., 2004; Gillan, Hartzell \& Parrino, 2009), ${ }^{10}$ and measures of the strength of social comparisons in an organization (Obloj and Zenger, 2017; Gartenberg and Wulf, 2017).

Moreover, the increased incidence of transparency (due to sunshine laws) may create a demand for field experiments in which organizations using subjective performance evaluation administer the pay structure predicted by our model to randomly selected employees, and allow the researchers to collect before-and-after data on employees' efforts. The results from this kind of experiment would allow organizations to gain insight on how to optimally respond to transparency laws.

\subsection{Optimal governance}

Having separately analyzed pay structure and incentive provision under secrecy and transparency, we now ask which of the two policies is optimal. We have shown that when the agents' efforts are verifiable, secrecy achieves the first best because it removes social comparison costs (Proposition 1). When efforts

\footnotetext{
${ }^{10}$ See Gil and Zanarone $(2017,2018)$ for a discussion of how the empirical literature on relational contracting has measured the value of collaborative relationships in organizations.
} 
and individual outputs are non-verifiable, however, the social comparison costs of transparency must be balanced against the firm's increased accountability towards employees. Which governance is optimal is therefore a priori ambiguous.

Proposition 5. With non-verifiable efforts, secrecy generates higher profits than transparency, and is therefore optimal, if bilateral employment relationships are strong enough (high $\delta$ ). Under weak employment relationships (low $\delta$ ), either transparency is optimal for all levels of social comparisons, or transparency is optimal if social comparisons are not too strong (low enough $\eta$ ), whereas secrecy is optimal if social comparisons are strong enough (high $\eta$ ).

\section{Proof. In Appendix A.}

If bilateral employment relationships are strong, the principal has enough credibility to persuade both agents to work hard under secrecy (formally, the bonus enforceability constraints (2) and (3) are not binding). In that case, secrecy is optimal because it removes social comparisons and the ensuing envy premium paid to agent 2. If employment relationships are weak, however, transparency has the advantage of making the principal's bonus payment promises more credible via multilateral enforcement. If social comparisons are mild (small $\eta$ ), their cost is more than compensated by the more balanced effort allocation and higher output induced by transparency. As $\eta$ grows larger, the balance of costs and benefits may be reversed and secrecy may become optimal. This needs not be the case, however, as the principal may prefer

to completely remove social comparisons by paying the envious agent a high enough rent $\left(w_{2}=\frac{\underline{u}_{1}}{1-\delta}\right)$. If effort distortions under secrecy are large relative to this rent, transparency may be optimal even under strong social comparisons.

Proposition 5 allows us to assess informal claims on the beneficial effects of transparency in the managerial literature on human resources. As a representative example consider Lawler (2000), who asserts: "There is a tremendous advantage to be gained from making pay rates and policies public. [...] Openness can increase trust, perception of fairness, understanding of the business, and respect for the 
organization and its management" (Lawler, 2000, p.287). Our model suggests an important sense in which transparency increases the employees' "trust" in the organization: by allowing multilateral enforcement, transparency enables the principal to use the stronger relationship with agent 2 to "subsidize" the weaker relationship with agent 1 . As a result, agent 1 is willing to trust higher bonus promises from the principal than he would under secrecy. Thus, transparency increases the overall level of "calculative" trust (Williamson, 1993) available in the organization and through that channel, enables the principal to allocate effort among the agents more efficiently. ${ }^{11}$

At the same time, our model does not support Lawler's (2000) claim that a switch from secrecy to transparency necessarily benefits the organization (i.e., increases profits). Due to social comparison costs, transparency may or may not be optimal. Thus, rather than looking for a "best practice", managers should make a piecemeal choice between secrecy and transparency, weighing features of the organization and of the social and institutional environment that favor one or the other policy. First, our model suggests that organizations should favor secrecy when the present discounted value of their relationships with individual employees is high because in that case, there are limited benefits from increasing employees' trust via transparency. This will be the case when the employment relationships are expected to be durable (i.e., the discount factor $\delta$ is high) or when output is expected to grow in the future. ${ }^{12}$ For instance, firms with a solid financial situation or operating in high-growth markets should choose secrecy to minimize social comparison costs. In contrast, firms facing a higher risk of bankruptcy or operating in satiated and crowded markets should choose transparency to boost their credibility vis-à-vis employees. This result also suggests that the optimal degree of transparency may vary over a firm's life cycle: firms face more uncertain

\footnotetext{
${ }^{11}$ A complementary dimension of trust, emphasized by sociological research and not modeled in our paper, is given by norms of reciprocity. These have been also shown to play an important governance role, especially in collaborative interfirm relationships (e.g., Granovetter, 1985; Gulati, 1995; Larson, 1992; Ring \& Van de Ven, 1992; Uzzi, 1997). Reciprocity has also been incorporated into formal models of incentive contracts (Englmaier and Leider, 2012), and relational contracts (Fahn, 2020).

${ }^{12}$ Output growth is not literally modeled in our stationary setup but it would be almost immediate to incorporate it into the model - for instance, by allowing for a positive probability that output permanently increases in the future.
} 
prospects, and therefore stand to gain more from boosting their own credibility via transparency, in their start-up phase than in the more mature stages of development.

A second implication of our model is that organizations should favor pay secrecy when social comparisons between employees, measured by $\eta$, are strong. This is more likely to be the case when the organization operates within an egalitarian culture, and when it features high geographical and social proximity between employees (Obloj and Zenger, 2017; Gartenberg and Wulf, 2017).

Lastly, our model suggests that organizations should favor secrecy over transparency when SPE is not necessary to motivate employees to exert effort (Proposition 1). This will be the case if employees' individual efforts or individual outputs can be objectively measured and, consequently, contractual outputbased incentives are effective. At the same time, even if individual outputs can be measured, our model suggests that organizations should choose transparency over secrecy when institutions are such that courts cannot be relied upon to enforce formal incentive contracts.

This last prediction could potentially be tested by looking at how a company's transparency responds to variations in the reliability of courts. Variation in contract enforceability and court quality has been exploited by recent studies of contracting both across countries (Antras and Foley, 2015) and within countries (Michler and Wu, 2020). Moreover, surveys such as that conducted every year by the US Chamber of Commerce show significant within-country variation in court quality, which has also been explored in academic research (e.g., Berkowitz and Clay, 2006). While a causal test of our prediction along these lines is beyond the scope of this paper, preliminary investigation of U.S. data does show a negative correlation between companies' transparency policies and court quality. 
Figure 4: Distribution of "transparency heroes" across US states by court quality

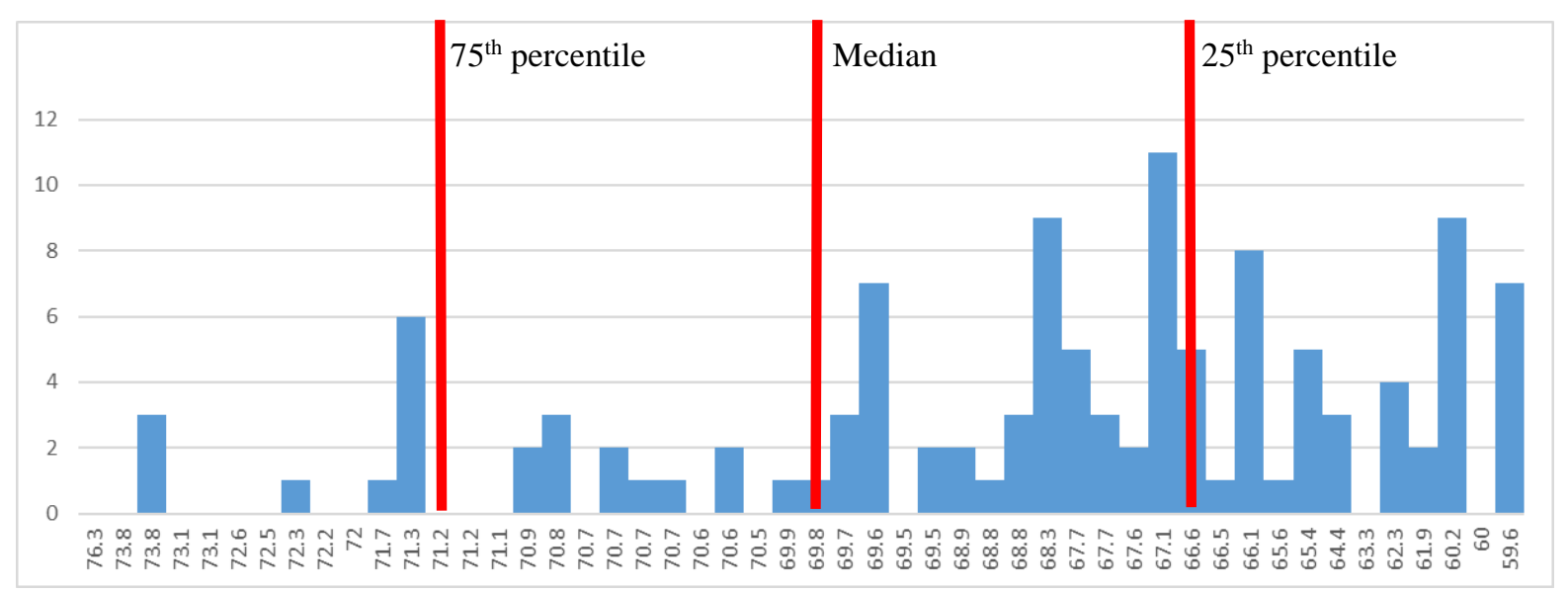

Figure 4 above shows the distribution of "transparency heroes" (companies that at least $40 \%$ of employees, surveyed by PayScale in 2017, classify as being highly transparent on pay) by the quality of courts in the U.S. state in which these companies or their U.S. subsidiaries are headquartered. The horizontal axis reports the court quality index that the US Chamber of Commerce assigned to each state in its 2019 Lawsuit Climate Survey. ${ }^{13}$ This index ranges from a minimum of 59.6 (Illinois) to a maximum of 76.3 (Delaware); Washington is the median state, while Oklahoma and Pennsylvania are the $75^{\text {th }}$ percentile and $25^{\text {th }}$ percentile state, respectively. The graph shows that $80 \%$ of transparency heroes are headquartered in states with below-the-median court quality (about $40 \%$ are headquartered in states with court quality below the $25^{\text {th }}$ percentile).

\footnotetext{
${ }^{13}$ The PayScale survey report is available at www.payscale.com/hero-awards/pay-transparency. The 2019 Lawsuit Climate Survey is available at www.instituteforlegalreform.com/uploads/pdfs/2019_Harris_Poll_State_Lawsuit_Climate_Ranking the_States.pdf.
} 
Figure 5: Who are the greatest transparency heroes?

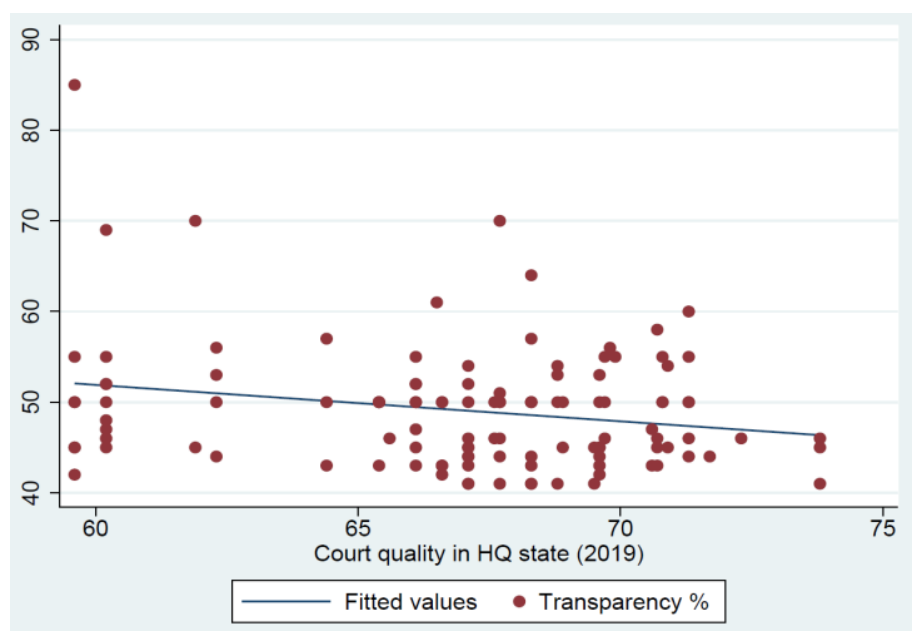

Figure 5 shows that even within the "transparency hero" group, the share of employees that classify a company as transparent, which ranges from $40 \%$ to $85 \%$ in the PayScale survey, is negatively correlated with court quality in the headquarter state. Regression analyses, available upon request, show that this correlation is robust to controlling for industry fixed effects and statistically significant at the 5\% level.

Altogether, the negative correlation between pay transparency and court quality is consistent with our prediction that transparency is more desirable when formal incentive contracts cannot be enforced.

\subsection{The effects of transparency laws}

In addition to elucidating the choice between transparency and secrecy, our model provides insight on the impact of "sunshine laws" on companies that rely on subjective performance evaluation to motivate their employees. Guided by public interest considerations, such as reducing pay discrimination based on gender and race, these laws impose transparency on organizations that were keeping pay information secret prior to their enactment.

It follows directly from Proposition 5 that when bilateral employment relationships or social comparisons are strong enough, such that secrecy is optimal, transparency laws reduce firm profits. Our next proposition shows the effect of transparency laws on the agents' efforts, output, and compensation. 
Proposition 6: Suppose secrecy is optimal. Then, transparency laws have the following effects. (1) They induce the organization to increase the salary of agent $2\left(w_{2}\right)$. (2) If secrecy elicits first best efforts, they do not affect effort and output. (3) If secrecy does not elicit first best efforts, they induce an efficient reallocation of effort from agent 2 to agent 1 and therefore increase output. Moreover, in that case, transparency laws induce the organization to increase the bonus of agent $1\left(b_{1}\right)$, and decrease the bonus of agent $2\left(b_{2}\right)$.

\section{Proof. In Appendix A.}

The case in which secrecy is optimal but does not elicit first best efforts occurs when bilateral employment relationships are weak and social comparisons are strong enough. In that case, under secrecy agent 1 exerts lower effort than agent 2 because he has a weaker bilateral relationship with the principal (Proposition 2). Once the law forces the firm to switch to transparency, the principal's credibility is enhanced by multilateral enforcement and the restructuring of the pay of agent 2 (Proposition 4). Taking advantage of her increased credibility, the principal reallocates effort from agent 2 to agent 1 by raising the latter's bonus (which is efficient because efforts have decreasing marginal productivities). This reallocation of effort increases output, and hence the organization's productive efficiency, although it reduces profits due to the rent the principal pays to the envious agent. This effect of transparency on efforts, output and compensation disappears when employment relationships are strong enough, such that both secrecy and transparency elicit first best efforts.

Note that once transparency laws are enacted, the gap between compensation, effort cost and the outside option is zero under both secrecy and transparency for agent 1 , whereas for agent 2 , this gap is higher under transparency than under secrecy, due to the envy premium. This implication of our model is seemingly inconsistent with recent evidence by Obloj and Zenger (2020), who find that transparency laws reduce the gap between the pay of US academics and the wage predicted by their rank, research outputs, experience, and affiliation. Obloj and Zenger (2020) interpret this evidence as suggesting that transparency reduces pay "inequity". While it is possible that employees may suffer not only from envy (inequality aversion) as 
modeled here, but also from inequity aversion, it is also important to notice that the employment setting analyzed by Obloj and Zenger (2020) is very different from ours, as pay in academia is typically contingent on objective output (e.g., publications) rather than SPE. ${ }^{14}$ Thus, a simple explanation for the empirical findings in Obloj and Zenger (2020) is that pay transparency may induce universities to make pay less sensitive to realized output to reduce envious social comparisons, and that, in turn, makes individual pay levels more closely aligned to the benchmarks predicted by rank, experience, and affiliation. Indeed, Obloj and Zenger (2020) also find that transparency laws reduce the sensitivity of pay to realized research output.

In our SPE model, incentive pay is conditional on inputs (the agents' effort) rather than output, and the credibility problem connected to the use of SPE creates a reason for increasing "pay inequity," which is absent in the setting analyzed by Obloj and Zenger (2020). Further empirical research is needed to assess whether the predictions of our model on the effect of transparency law on pay structures are borne out in settings where SPE is used.

\section{Extensions and Robustness}

\subsection{Exogenous information on peer's effort}

In our baseline model we have assumed transparency makes both an agent's pay and his effort observable to his peer. In this section we explore the alternative scenario in which effort observability by peers is a fixed feature of the environment that cannot be modified by policy.

We begin with the case in which effort is perfectly observable by peers under both secrecy and transparency. Under this assumption, our analysis of pay structure and efforts under transparency (Propositions 3 and 4) continues to apply. However, the analysis of secrecy changes because now the principal can only prevent agents from observing each other's pay so even under secrecy, the agents may

\footnotetext{
${ }^{14}$ Tenure and promotion decisions, on the other hand, are partly contingent on subjectively assessed faculty inputs such as administrative service, professionalism, and "good citizenship".
} 
suffer from social comparisons due to unequal efforts. Thus, consistent with our view that agents engage in self-serving beliefs - that is, they base social comparisons only on visible payoff differences - we assume that when efforts are visible but pay is secret, an agent's social comparison disutility is:

$$
u_{i} \equiv w_{i}+b_{i}-c\left(e_{i}\right)-\eta \max \left\{0, c\left(e_{i}\right)-c\left(e_{j}\right)\right\}, \text { for } i, j \in\{1,2\} .
$$

The analysis is similar to section 4.1 , except that now agent 2 , who has a stronger relationship with the principal and hence is required to exert more effort, can avoid social comparison costs by shirking. Thus, relative to section 4.1, the principal must pay a higher incentive bonus to induce agent 2 to exert a given effort, implying that when the principal's enforceability constraint is binding, the profit from her relationship with agent 2 is smaller than before. As a result, secrecy becomes less profitable when the principal cannot prevent agents from observing each other's efforts. Nevertheless, the tradeoff between secrecy and transparency illustrated by Proposition 5 continues to exist.

Proposition 7. When agents can observe each other's effort, secrecy generates lower efforts and profits than when the effort levels are kept private. Nevertheless, secrecy continues to be optimal under strong employment relationships (high enough $\delta$ ).

Proof: in appendix A.

The intuition behind this result is simple. When employment relationships are strong enough, the principal can elicit first best efforts from both agents. Since pay is secret, the fact that both agents exert the same effort removes social comparisons, thus making secrecy optimal.

Consider now the opposite case in which an agent's effort is unobservable by his peer under both secrecy and transparency and therefore does not give rise to social comparisons. When efforts are invisible but pay is transparent, an agent's utility is:

$$
u_{i} \equiv w_{i}+b_{i}-c\left(e_{i}\right)-\eta \max \left\{0, w_{j}+b_{j}-w_{i}-b_{i}\right\}, \text { for } i, j \in\{1,2\} .
$$

In this case, the analysis of secrecy is identical to section 4.1. The analysis of transparency, though, is more subtle. We discuss some of the key issues and their underlying intuition here, while referring readers to Appendix B for the details and a formal analysis. First, unobservability of peer's efforts potentially 
complicates multilateral enforcement. If agent 1 interprets non-payment of the promised bonus to agent 2 as a defection and punishes the principal accordingly, agent 2 may take advantage of that and fail to exert effort in the hope to nevertheless receive the bonus. Notice, however, that the principal would only have an incentive to do so if the future relationship with agent 2 was so valuable to render payment of both bonuses optimal. Here, we therefore abstract from such issues and assume that each agent receives the bonus if, and only if she exerts effort. Under this assumption, the trade-off between secrecy and transparency illustrated by Proposition 5 continues to hold: If the value of employment relationships (measured by $\delta$ ) is sufficiently high, secrecy is optimal because it delivers first-best outcomes without social comparisons. If social comparisons (measured by $\eta$ ) are weak, transparency is optimal because it provides the benefits of multilateral enforcement without triggering too pronounced social comparison costs.

Second, whereas the mechanisms that drove optimal pay and effort allocation in section 4 are still present, the fact that efforts are not observable to peers creates additional tradeoffs. Pay differences among the agents now generate higher social comparison costs because they are no longer "moderated" by opposite differences in effort costs. To partially compensate the higher premium she must now pay to the envious agent (i.e., agent 2), the principal will raise his effort, and lower the effort and pay of agent 1, relative to the baseline model, taking advantage of the fact that changes in effort levels no longer affect social comparisons. As a result, under strong social comparisons it may be optimal for the principal to have $e_{2}>$ $e_{1}$, and in that case an important advantage of transparency over secrecy - namely, ensuring a more balanced allocation of effort among agents - will be reduced.

Moreover, our result that the pay of agent 2 is biased towards the fixed salary (formally, the salary is larger than the agent's social comparison cost, and the bonus is smaller than his effort cost) is now true regardless the strength of social comparisons (in Section 4, instead, this result only holds if $\eta$ is sufficiently large). Again, this follows from the fact that when efforts are not observable to peers, social comparisons are not moderated by effort costs, and thus the principal must address them more vigorously through the design of the agent's pay structure. The model with unobservable effort has a few additional interesting 
theoretical implication - for instance, for the compensation structure of agent 1 . We refer readers interested in a full analysis to Appendix B.

Altogether, our analysis shows that when the agents' information on each other's effort is fixed, one of the two policies performs worse than when such information is chosen by the principal. This suggests that organization design, particularly to what extent the organization makes performance evaluations transparent and promotes information sharing among employees, importantly complements pay policy as a means to solve the tradeoff between accountability and social comparison costs. The benefits of secrecy (transparency) are maximized by a corporate culture in which performance evaluations are private (open), and employees are discouraged from discussing (encouraged to discuss) performance evaluation and compensation with each other.

\subsection{Symmetric inequality aversion}

Motivated by a growing body of empirical evidence, our baseline model assumes the agents are envious, that is, they only suffer when their payoff is lower than their peer's one. In this section we briefly consider the case in which agents are more broadly averse to inequality (but abstain from a complete formal characterization of results), in the sense that they also suffer when their payoff is higher than their peer's (e.g., Grund and Sliwka, 2005; Englmaier and Wambach, 2010). To analyze this case, we add to our model a "compassion" parameter $\alpha \leq \eta$, such that an agent's total social comparison disutility is now given by:

$$
\begin{aligned}
& u_{i} \equiv w_{i}+b_{i}-c\left(e_{i}\right)-\eta \max \left\{0, m_{j}-m_{i}\right\}-\alpha \max \left\{0, m_{i}-m_{j}\right\}, \text { for } i, j \in\{1,2\}, \\
& \text { where } m_{i}=w_{i}+b_{i}-c\left(e_{i}\right) \text { is the "material payoff" of agent } i \text {, as usual. }
\end{aligned}
$$

The analysis of secrecy is the same as section 3.1. The analysis of transparency is very similar to section 4.2, except that now both agent 2 and agent 1 suffer from social comparisons and therefore the principal has a stronger incentive than before to equalize the agents' material payoffs by paying agent 2 a rent, with agent 2's compensation structure as before (a salary higher than his social comparison disutility, a bonus lower than the effort cost). In that case, social comparisons due to compassion can even benefit the principal 
because they reduce agent 2's incentive to shirk: if he shirked, agent 2 would now have a higher material payoff than agent 1 because of his high salary, and that would make him feel sorry for agent 1 and hence suffer a disutility. Altogether, the additional presence of compassion can make transparency more profitable than in the case in which only envy is present, although the tradeoff illustrated by Proposition 5 continues to exist.

\section{Conclusion}

This paper has developed a formal theory of the costs and benefits of pay transparency for organizations that incentivize their employees through subjective performance assessments. We have shown that transparency increases the employees' ability to jointly hold their organization accountable through a threat of "multilateral enforcement". Increased accountability, in turn, makes the promise of subjective incentive bonuses more credible, allowing the organization to more flexibly allocate effort among its employees. At the same time, we have shown that transparency facilitates envious social comparisons among employees, which require the organization to restructure the employees' pay in a way that increases the weight of fixed compensation relative to subjective bonuses. We have discussed several implications of our model for the choice between pay transparency and secrecy, compensation design, and the effect of transparency laws on compensation and employees' efforts.

We hope our paper will stimulate future research on the important, yet understudied topic of how pay transparency affects organizations. In particular, future work may exploit exogenous variations in pay transparency due to legislative interventions to test the predictions of our model, and compare the effects of transparency in firms that do or do not subjective performance evaluation. Rather than advocating a onesize-fits all policy, the line of research opened by our paper holds the promise to inform managers on when to pursue transparency, and how to optimally adapt the organization's incentive system to it. 


\section{References}

Adams, JS., (1963), “Toward an understanding of inequity,” Journal of Abnormal and Social Psychology 67(5): 422-436.

Akerlof, G., and J. Yellen, (1990), “The fair wage-effort hypothesis and unemployment," Quarterly Journal of Economics 105: 255-283.

Antras, P., and F. Foley, (2015), "Poultry in Motion: A Study of International Trade Finance Practices," Journal of Political Economy, 123: 809-852.

Argyres, N., J. Bercovitz, and G. Zanarone, (2020), “The Role of Relationship Scope in Sustaining Relational Contracts in Interfirm Networks,” Strategic Management Journal 41: 222-245.

Baker, G., R. Gibbons, and K. J. Murphy, (1994), "Subjective Performance Measures in Optimal Incentive Contracts," Quarterly Journal of Economics, 109(4), 1125-1156.

Baker, G., R. Gibbons, and K. J. Murphy (2002), "Relational Contracts and The Theory Of The Firm," Quarterly Journal of Economics 117: 39-84.

Baker, G., R. Gibbons, and K. J. Murphy (2011), "Relational Adaptation,” MIT Sloan working paper.

Bamberger, P., and E. Belogolovsky, (2010), “The Impact of Pay Secrecy on Individual Task Performance," Personnel Psychology 63: 965-996.

Barron, D., R. Gibbons, R. Gil, and K. J. Murphy, (2019), “Relational Adaptation under Reel Authority," forthcoming in Management Science.

Belogolovsky, E., and P. Bamberger, (2014), "Signaling in Secret: Pay for Performance and the Incentive and Sorting Effects of Pay Secrecy," Academy of Management Journal 57: 1706-1733.

Bercowitz, D., and K. Clay, (2006), “The effect of judicial independence on courts: Evidence from the American states," Journal of Legal Studies 35: 399-440. 
Breza, E., S. Kaur, and Y. Shamdasani, (2018), “The Morale Effects of Pay Inequality,” Quarterly Journal of Economics 133: 611-633.

Cao, Z., and F. Lumineau, (2015), "Revisiting the Interplay between Contractual and Relational Governance: A Qualitative and Meta-Analytic Investigation," Journal of Operations Management 33-34: $15-42$.

Card, D., A. Mas, E. Moretti, and E. Saez, (2012), "Inequality at Work: The Effect of Peer Salaries on Job Satisfaction," American Economic Review 102: 2981-3003.

Cohn, A., E. Fehr, B. Hermann, and F. Schneider, (2014), "Social Comparison and Effort Provision: Evidence from a Field Experiment," Journal of the European Economic Association 12: 877-898.

Colella, A., R. Paetzold, A. Zardkoohi, and M. Wesson, (2007), "Exposing Pay Secrecy," Academy of Management Review 32: 55-71.

Contreras, O., M. Fahn, and G. Zanarone, (2019), "Managing Social Comparisons in Organizations," working paper.

Cullen, Z., and Pakzad-Hurson, B., (2020), “Equilibrium Effects of Pay Transparency in a Simple Labor Market," Harvard Business School working paper.

Cullen, Z., and R. Perez-Truglia, (2019), “The Salary Taboo: Privacy Norms and the Diffusion of Information," working paper.

Dur, R., and J. Tichem (2015), “Altruism and Relational Incentives in the Workplace,” Journal of Economics and Management Strategy 24: 485-500.

Edelman, B., and I. Larkin, (2014), "Social Comparisons and Deception across Workplace Hierarchies: Field and Experimental Evidence," Organization Science 26: 78-98. 
Edwards, M., (2005), “The Law and Social Norms of Pay Secrecy,” Journal of Employment and Labor Law 26: 41-63.

Englmaier, F., and A. Wambach, (2010), "Optimal incentive contracts under inequity aversion," Games and Economic Behavior, 69: 312-328.

Englmaier, F., and S. Leider, (2012), “Contractual and Organizational Structure with Reciprocal Agents," American Economic Journal: Microeconomics 4: 146-183.

Fahn, M. (2020), “Reciprocity in Dynamic Employment Relationships,” Working Paper.

Fahn, M., A. Schade, and K. Schuessler, (2017), "What Drives Reciprocal Behavior? The Optimal Provision of Incentives over the Course of Careers," Working Paper.

Fehr, E., and K. Schmidt, (1999), “A Theory of Fairness, Competition, and Cooperation,” Quarterly Journal of Economics 114: 817-868.

Feldman, E., C. Gartenberg, and J. Wulf, (2018), “Pay Inequality and Corporate Divestitures," Strategic Management Journal 39: 2829-2858.

Foss N., (2003), "Selective intervention and internal hybrids: interpreting and learning from the rise and decline of the Oticon spaghetti organization," Organization Science 14:331-49.

Frederiksen, A., F. Lange, and B. Kriechel, (2017), “Subjective performance evaluations and employee careers," Journal of Economic Behavior and Organization 134: 408-429.

Futrell, C., (1978), "Effects of Pay Disclosure on Satisfaction for Sales Managers," Academy of Management Journal 21: 140-144.

Gartenberg, C., and J. Wulf, (2017), "Pay Harmony? Social Comparison and Performance Compensation in Multibusiness Firms," Organization Science 28: 39-55. 
Gartenberg, C., and J. Wulf, (2019), "Competition and Pay Inequality Within and Between Firms," forthcoming in Management Science.

Gely, R., and L. Bierman, (2003), "Pay Secrecy Confidentiality Rules and the National Labor Relations Act," University of Pennsylvania Journal of Labor and Employment Law 6: 122-156.

Gibbs, M., K. Merchant, W. Van der Stede, and M. Vargus, (2004), "Determinants and Effects of Subjectivity in Incentives," Accounting Review 79: 409-436.

Gil, R., M. Kim, and G. Zanarone, (2021), "Relationships Under Stress: Relational Outsourcing in the US Airline Industry After the 2008 Financial Crisis," forthcoming in Management Science.

Gil, R., and G. Zanarone, (2017), “Formal and Informal Contracting: Theory and Evidence,” Annual Review of Law and Social Science 13: 141-159.

Gil, R., and G. Zanarone, (2018), "On the Determinants and Consequences of Informal Contracting," Journal of Economics and Management Strategy 27: 726-741.

Gillan, S., J. Hatzell, and R. Parrino, (2009), “Explicit Versus Implicit Contracts: Evidence from CEO Employment Agreements," Journal of Finance 64: 1629-55.

Gino, F., and L. Pierce, (2009), “The Abundance Effect: Unethical Behavior in the Presence of Wealth," Organizational Behavior and Human Decision Processes 109: 142-155.

Gino, F., and L. Pierce, (2010), "Lying to Level the Plain Field: Why People May Dishonestly Help or Hurt Others to Create Equity," Journal of Business Ethics 95: 89-103.

Granovetter, M., (1985), “Economic Action and Social Structure: The Problem of Embeddedness,” American Journal of Sociology 91: 481-510.

Greif, A., (1994), "Cultural Beliefs and the Organization of Society: A Historical and Theoretical Reflection on Collectivist and Individualist Societies," Journal of Political Economy 102: 912-950. 
Grund, C., and D. Sliwka, "Envy and Compassion in Tournaments, (2005)," Journal of Economics and Management Strategy 14: 187-207.

Gulati, R., (1995), "Social Structure and Alliance Formation Patterns: A Longitudinal Analysis," Administrative Science Quarterly 40: 619-652.

Hastings, R., and E. Meyer, (2020), No Rules Rules: Netflix and the Culture of Reinvention, New York: Penguin Press.

Hayes, R., and S. Schaefer, (2004), "Implicit Contracts and the Explanatory Power of Top Executive Compensation for Future Performance," RAND Journal of Economics 31: 273-293.

Hill, A., (2016), "Pay Transparency is the Last Taboo in Business," Financial Times (September 19).

Kampkotter, P., and D. Sliwka, (2015), “The Complementary Use of Experiments and Field Data to Evaluate Management Practices: The Case of Subjective Performance Evaluations," IZA Discussion Papers, No. 9285.

Kerr, S., (1975), “On the Folly of Rewarding A, while Hoping for B, Academy of Management Journal 18: 779-783.

Klein, B. (2000), “The Role of Incomplete Contracts in Self-Enforcing Relationships,” Revue d'Économie Industrielle 92: 67-80.

Kragl, J., and J. Schmid, (2009), “The impact of envy on relational employment contracts," Journal of Economic Behavior and Organization, 72(2), 766-779.

Kosova, R., and G. Sertsios, (2018), “An Empirical Analysis of Self-Enforcement Mechanisms: Evidence from Hotel Franchising," Management Science 64: 43-63.

Kunda, Z., (1990), “The Case for Motivated Reasoning,” Psychological Bulletin 108: 480-498. 
Larkin, I., L. Pierce, and F. Gino, (2012), “The Psychological Costs of Pay-for-Performance: Implications for the Strategic Compensation of Employees," Strategic Management Journal 33: 11941214.

Larson, A., (1992), "Network Dyads in Entrepreneurial Settings: A Study of Governance in Exchange Relationships,” Administrative Science Quarterly 37: 76-104.

Lawler, E., (2000), Rewarding excellence: Pay strategies for the new economy. San Francisco: Jossey-Bass.

Levin, J., (2002), “Multilateral Contracting and the Employment Relationship,” Quarterly Journal of Economics 117: 1075-1103.

Levin, J., (2003), “Relational Incentive Contracts,” American Economic Review 93: 835-857.

Michler, J., and S. Wu, (2020), "Governance and contract choice: Theory and evidence from groundwater irrigation markets," Journal of Economic Behavior and Organization 180: 129-147.

Nickerson, J. A., and T. R. Zenger, (2008), "Envy, comparison costs, and the economic theory of the firm," Strategic Management Journal, 29(13), 1429-1449.

Obloj, T., and T. Zenger, (2017), “Organization Design, Proximity, and roductivity Responses to Upward Social Comparison,” Organization Science 28: 1-18.

Obloj, T., and T. Zenger, (2020), “The Influence of Pay Transparency on Inequity, Inequality, and the Performance-Basis of Pay in Organizations," working paper.

Ockenfels, A., D. Sliwka, and P. Werner, (2015), "Bonus Payments and Reference Point Violations," Management Science 61: 1496-1513.

Poppo, L., and T. Zenger, (2002), "Do Formal Contracts and Relational Governance Function as Substitutes or Complements?" Strategic Management Journal 23: 707-25. 
Ring, P., and A. Van de Ven, (1992), "Structuring Cooperative Relationships between Organizations," Strategic Management Journal 13: 483-498.

Ryall, M., and R. Sampson, (2009), "Formal Contracts in the Presence of Relational Enforcement Mechanisms: Evidence from Technology Development Projects," Management Science 55: 906-25.

Shue, K., (2013), "Executive Networks and Firm Policies: Evidence from the Random Assignment of MBA Peers," Review of Financial Studies 26: 1401-1442.

Spagnolo, G., (1999), "Social Relations and Cooperation in Organizations," Journal of Economic Behavior and Organization 38: 1-25.

Stewart J., (1993), “Taking the dare,” New Yorker, July 26, pp. 34-39.

Uzzi, B., (1997), "Social Structure and Competition in Interfirm Networks," Administrative Science Quarterly 42: 35-67.

Wade, J., C. O’Reilly, and T. Pollock, (2006), “Overpaid CEOs and Underpaid Managers: Fairness and Executive Compensation," Organization Science 17: 527-544.

Williamson, O., (1993), “Calculativeness, Trust, and Economic Organization,” Journal of Law and Economics 36: 453-486.

Zanarone, G. (2013), “Contract Adaptation under Legal Constraints,” Journal of Law, Economics and Organization 29: 799-834. 


\section{Appendix A: Mathematical Proofs}

\section{Proof of Proposition 1.}

Under secrecy, the following contract (not uniquely) maximizes the principal's profits and lets both agents accept the contract and exert first-best effort: $w_{i}=c\left(e^{F B}\right)+\underline{u}_{i}$; if and only $e_{i}=e^{F B}$ (otherwise, $\left.w_{i} \leq 0\right)$. Then, the principal's profits are $\pi=y\left(e^{F B}, e^{F B}\right)-\underline{u}_{1}-2 c\left(e^{F B}\right)$, which is equivalent to the maximized surplus.

Under transparency, a wage $w_{i}$ again is only paid if agent $i$ exerts equilibrium effort, with a deviation punished by a sufficiently low compensation. Thus, the principal maximizes $\pi=y\left(e_{1}, e_{2}\right)-$ $w_{1}-w_{2}$, subject to the following participation constraints (PC):

$$
\begin{aligned}
& m_{1}-\max \left\{0, \eta\left(m_{2}-m_{1}\right)\right\} \geq \underline{u}_{1} \\
& m_{2}-\max \left\{0, \eta\left(m_{1}-m_{2}\right)\right\} \geq 0
\end{aligned}
$$

First, we show that $m_{1} \geq m_{2}$, hence agent 1 does not suffer from social comparison costs. To the contrary, assume there is a profit-maximizing equilibrium with $m_{1}<m_{2}$. Then, (PC2) must bind because otherwise, the principal could reduce $w_{2}$ without violating any constraint. Thus, $m_{2}=0$. However, this contradicts $m_{2}>m_{1}$ because (PC1) requires $m_{1} \geq \underline{u}_{1}>0$. Therefore, $m_{1} \geq m_{2}$ and, for the same reasons as just laid out, (PC1) must bind. This yields $w_{1}=c\left(e_{1}\right)+\underline{u}_{1}$, which we can plug into (PC2), $\left(w_{2}-c\left(e_{2}\right)\right)(1+\eta)-\eta \underline{u}_{1} \geq 0$. (PC2) must also bind because otherwise, the principal could reduce $w_{2}$ and thereby increase her profits. Thus, the principal chooses $e_{1}$ and $e_{2}$ to maximize

$$
\pi=y\left(e_{1}, e_{2}\right)-\underline{u}_{1}-c\left(e_{1}\right)-c\left(e_{2}\right)-\frac{\eta \underline{u}_{1}}{(1+\eta)}
$$


and first-order conditions are

$$
\begin{aligned}
& y_{1}-c^{\prime}\left(e_{1}\right)=0 \\
& y_{2}-c^{\prime}\left(e_{2}\right)=0 .
\end{aligned}
$$

Thus, $e_{1}=e_{2}=e_{1}^{F B}$.

It follows that profits under transparency are smaller than under secrecy. Both implement the same effort, however agent 2's compensation under transparency is smaller because it must contain a social comparison premium.

\section{Proof of Proposition 2.}

Note that for production to potentially be optimal, we need to impose an assumption that the principal's profits are positive in case she appropriates the entire surplus and $e^{F B}$ is implemented, hence

$$
y\left(e^{F B}\right)-c\left(e^{F B}\right)-\underline{u}_{1}>0
$$

must hold. For agent 2, this condition is always satisfied since $c^{\prime}(0)=0$ and $y^{\prime}>0$.

Now, due to stationarity, the principal's problem is to maximize

$$
\pi=y\left(e_{1}, e_{2}\right)-w_{1}-w_{2}-b_{1}-b_{2}
$$

in every period, subject to the constraints

$$
\begin{aligned}
& \frac{w_{i}+b_{i}-c\left(e_{i}\right)}{1-\delta} \geq \frac{\underline{u}_{i}}{1-\delta} \\
& b_{i}-c\left(e_{i}\right)+\delta \frac{w_{i}+b_{i}-c\left(e_{i}\right)}{1-\delta} \geq \delta \frac{\underline{u}_{i}}{1-\delta} \\
&-b_{i}+\delta \frac{y\left(e_{i}\right)-w_{i}-b_{i}}{1-\delta} \geq 0,
\end{aligned}
$$


for $i \in\{1,2\}$, and where (PC) stands for participation constraint, (IC) for incentive constraint, and (EC) for enforceability constraint. The (PC) constraints make sure that both agents find it optimal to work for the principal, compared to rejecting her offer and consuming their outside options. The (IC) constraints state that exerting equilibrium effort is optimal for each agent. We assume that, if an agent deviates and chooses an effort level different from $e_{i}$, he does not receive the bonus $b_{i}$ and the relationship is subsequently terminated. Therefore, if an agent deviates, he deviates to an effort level of zero. Finally, the (EC) constraints are necessary because effort is not verifiable. Therefore, paying the bonus to agent $\mathrm{i}$ is only optimal for the principal if future discounted profits in the relationship with agent $\mathrm{i}$ are high enough.

First, we show that there is a profit-maximizing equilibrium in which (PCi) and (ICi) hold as equalities: To the contrary, assume that (ICi) is slack. If $b_{i}>0$, the principal can reduce $b_{i}$ by a small $\varepsilon>0$ and increase $w_{i}$ by $\varepsilon$. This keeps (PCi) and $\pi_{i}=y\left(e_{i}\right)-w_{i}-b_{i}$ unaffected, but relaxes (ECi). If $b_{i}=0$, the principal can reduce $w_{i}$ by a small $\varepsilon>0$ without violating any constraint (for $b_{i}=0$, (ICi) is tighter than (PCi)). A binding (IC) yields $b_{i}=c\left(e_{i}\right)-\delta\left(w_{i}-\underline{u}_{i}\right)$, thus (PCi) becomes $w_{i} \geq \underline{u}_{i}$. It has to bind because, if it is slack, the principal can reduce $w_{i}$ by a small $\varepsilon>0$ and increase $b_{i}$ by $\delta \varepsilon$ to keep (ICi) unaffected. This also keeps (DE) unaffected but increases $\pi_{i}$.

Binding (PCi) and (ICi) constraints yield $w_{i}+b_{i}-c\left(e_{i}\right)=\underline{u}_{i}$ and $b_{i}=c\left(e_{i}\right)$, proving the first part of the proposition. Taking this into account, the optimization problem becomes to maximize

$$
\pi=y\left(e_{1}, e_{2}\right)-c\left(e_{1}\right)-c\left(e_{2}\right)-\underline{u}_{1},
$$

subject to

$$
\begin{aligned}
-c\left(e_{1}\right)+\delta\left(y\left(e_{1}\right)-\underline{u}_{1}\right) & \geq 0 \\
-c\left(e_{2}\right)+\delta y\left(e_{2}\right) & \geq 0 .
\end{aligned}
$$

Now, we show that there exist thresholds $\bar{\delta}_{i} \in(0,1)$, with $\bar{\delta}_{2}<\bar{\delta}_{1}$, such that $e_{i}=e^{F B}$ if $\delta \geq \bar{\delta}_{i}$, whereas $e_{i}<e^{F B}$ for $\delta<\bar{\delta}_{i}$. In the latter case, $e_{2}$ is determined by the binding (EC2) constraint, 
whereas $e_{1}$ is either determined by the binding (EC1) constraint, or $e_{1}=0$. It is immediate that (ECi) constraints hold for $e^{F B}$ if $\delta \rightarrow 1$. To establish existence of the threshold $\bar{\delta}_{1}$, fix any effort level $\hat{e}_{1}<e_{1}^{F B}$ such that $y\left(\hat{e}_{1}\right)-c\left(\hat{e}_{1}\right)-\underline{u}_{1}>0$. Then, the left hand side of (EC1) increases in $\delta$, and (EC1) is satisfied for $\delta$ sufficiently large. If $y\left(\hat{e}_{1}\right)-c\left(\hat{e}_{1}\right)-\underline{u}_{1} \leq 0$, production is not profitable with $\hat{e}_{1}$ (note that, in the following, we mostly assume that positive effort levels can be implemented for agent 1 under secrecy). Thus, there exists a threshold $\bar{\delta}_{1}$ with the properties just described. It follows that the same holds for (EC2) and $\bar{\delta}_{2}$, establishing the second part of the proposition. Regarding its third part, note that $\bar{\delta}_{1}>\bar{\delta}_{2}$ (and $e_{1}<e_{2}$ for $\delta<\bar{\delta}_{1}$ ) is implied by $\underline{u}_{1}>0$ and the concavity of $y(\cdot)$.

\section{Proof of Proposition 3.}

The principal maximizes

$$
\pi=y\left(e_{1}, e_{2}\right)-w_{1}-w_{2}-b_{1}-b_{2}
$$

in every period, subject to the following constraints.

$$
\begin{aligned}
w_{1}+b_{1}-c\left(e_{1}\right)-\max \left\{0, \eta\left(m_{2}-m_{1}\right)\right\} & \geq \underline{u}_{1} \\
w_{2}+b_{2}-c\left(e_{2}\right)-\max \left\{0, \eta\left(m_{1}-m_{2}\right)\right\} & \geq 0 \\
b_{1}-c\left(e_{1}\right)-\max \left\{0, \eta\left(m_{2}-m_{1}\right)\right\}+\delta \frac{u_{1}}{1-\delta} & \geq-\max \left\{0, \eta\left(m_{2}-w_{1}\right)\right\}+\delta \frac{\underline{u}_{1}}{1-\delta} \\
b_{2}-c\left(e_{2}\right)-\max \left\{0, \eta\left(m_{1}-m_{2}\right)\right\}+\delta \frac{u_{2}}{1-\delta} & \geq-\max \left\{0, \eta\left(m_{1}-w_{2}\right)\right\} \\
-b_{1}-b_{2}+\delta \frac{\pi}{1-\delta} & \geq 0
\end{aligned}
$$




\section{Preliminaries}

This optimization problem can be substantially simplified. First, we show that $m_{1}-m_{2} \geq 0$. To the contrary, assume $m_{2}>m_{1}$, in which case the set of constraints would be

$$
\begin{aligned}
w_{1}+b_{1}-c\left(e_{1}\right)-\eta\left(m_{2}-m_{1}\right) & \geq \underline{u}_{1} \\
w_{2}+b_{2}-c\left(e_{2}\right) & \geq 0 \\
b_{1}-c\left(e_{1}\right)+\max \left\{0, \eta\left(m_{2}-w_{1}\right)\right\}-\eta\left(m_{2}-m_{1}\right)+\delta \frac{u_{1}}{1-\delta} & \geq \delta \frac{\underline{u}_{1}}{1-\delta} \\
b_{2}-c\left(e_{2}\right)+\max \left\{0, \eta\left(m_{1}-w_{2}\right)\right\}+\delta \frac{u_{2}}{1-\delta} & \geq 0 \\
-b_{1}-b_{2}+\delta \frac{\pi}{1-\delta} & \geq 0
\end{aligned}
$$

Note that $m_{2}>m_{1}$ implies $w_{2}+b_{2}-c\left(e_{2}\right)>\underline{u}_{1}$, hence (PC2) is slack. If (IC2) is also slack, the principal can reduce $w_{2}$ until either (IC2) binds or $m_{2}=m_{1}$. This operation relaxes (PC1); it either relaxes (IC1) (if $m_{2}-w_{1}<0$ ) or keeps it unaffected (if $m_{2}-w_{1} \geq 0$ ), whereas (EC) is relaxed and profits go up. If (IC2) binds, the principal can reduce $w_{2}$ by a small $\varepsilon>0$ and increase $b_{2}$ by $\delta \varepsilon$. This operation either leaves (IC2) unaffected (if $m_{1}-w_{2}<0$ ) or relaxes it (if $m_{1}-w_{2} \geq 0$ ). Moreover, (PC1) is relaxed, (IC1) is either relaxed (if $m_{2}-w_{1}<0$ ) or unaffected (if $m_{2}-w_{1} \geq 0$ ), whereas (EC) is unaffected but profits go up. Thus, $m_{2}>m_{1}$ cannot be optimal, and $m_{1} \geq m_{2}$.

This yields the following set of constraints:

$$
\begin{aligned}
w_{1}+b_{1}-c\left(e_{1}\right) & \geq \underline{u}_{1} \\
w_{2}+b_{2}-c\left(e_{2}\right)-\eta\left(m_{1}-m_{2}\right) & \geq 0 \\
b_{1}-c\left(e_{1}\right)+\max \left\{0, \eta\left(m_{2}-w_{1}\right)\right\}+\delta \frac{u_{1}}{1-\delta} & \geq \delta \frac{\underline{u}_{1}}{1-\delta} \\
b_{2}-c\left(e_{2}\right)-\eta\left(m_{1}-m_{2}\right)+\max \left\{0, \eta\left(m_{1}-w_{2}\right)\right\}+\delta \frac{u_{2}}{1-\delta} & \geq 0 \\
-b_{1}-b_{2}+\delta \frac{\pi}{1-\delta} & \geq 0
\end{aligned}
$$

In the next step we show that there is a profit-maximizing equilibrium in which (PC1) and (IC1) 
bind. To the contrary, assume that either (PC1) or (IC1) is slack. First, assume that (PC1) is slack and (IC1) binds. Then, the principal can reduce $w_{1}$ by $\varepsilon$ and increase $b_{1}$ by $\delta \varepsilon$. This operation relaxes (PC2), whereas (IC1) and (IC2) either are unaffected or relaxed. Finally, (EC) is unaffected and profits go up. Second, assume that (PC1) binds and (IC1) is slack. Then, the principal can increase $w_{1}$ by $\varepsilon$ and reduce $b_{1}$ by $\varepsilon$. This operation relaxes (EC) and does not violate any other constraint if $\varepsilon$ is sufficiently small. Third, assume that (PC1) and (IC1) are slack. Then, the principal can reduce $b_{1}$ until either of them binds.

Thus, there is profit-maximizing equilibrium in which (PC1) and (IC1) bind, which yields $b_{1}=$ $c\left(e_{1}\right)-\max \left\{0, \eta\left(m_{2}-w_{1}\right)\right\}$ and $w_{1}=\underline{u}_{1}+\max \left\{0, \eta\left(m_{2}-w_{1}\right)\right\}$

In the next step, we show that $m_{2}-w_{1} \leq 0$. To the contrary, assume $m_{2}-w_{1}>0$. Then, $b_{1}=$ $c\left(e_{1}\right)-\eta \frac{m_{2}-\underline{u}_{1}}{1+\eta}$ and $w_{1}=\frac{\underline{u}_{1}+\eta m_{2}}{1+\eta}$, and $m_{2}-w_{1}>0$ becomes $m_{2}-\underline{u}_{1}>0$. Since $m_{1}=\underline{u}_{1}$, this condition is inconsistent with $m_{1} \geq m_{2}$.

Thus, $m_{2}-w_{1} \leq 0$, hence $b_{1}=c\left(e_{1}\right)$ and $w_{1}=\underline{u}_{1}$, which will further be used in the proof to Proposition 4.

\section{Main Analysis}

Collecting the previously derived results, the optimization problem becomes to maximize

$$
\pi=y\left(e_{1}, e_{2}\right)-\underline{u}_{1}-w_{2}-c\left(e_{1}\right)-b_{2}
$$

in every period, subject to the following constraints.

$$
\begin{aligned}
\left(w_{2}+b_{2}-c\left(e_{2}\right)\right)(1+\eta)-\eta \underline{u}_{1} & \geq 0 \\
\left(w_{2}+b_{2}-c\left(e_{2}\right)\right)(1+\eta)-\eta \underline{u}_{1} & \geq(1-\delta)\left[w_{2}-\max \left\{0, \eta\left(\underline{u}_{1}-w_{2}\right)\right\}\right] \\
-c\left(e_{1}\right)-b_{2}+\delta \frac{y\left(e_{1}, e_{2}\right)-c\left(e_{1}\right)-\underline{u}_{1}-w_{2}-b_{2}}{1-\delta} & \geq 0 .
\end{aligned}
$$

Moreover, we have to take account of the consistency requirement $m_{1} \geq m_{2}$. 
In the following we distinguish between A) $\underline{u}_{1}>w_{2}$, and B) $\underline{u}_{1} \leq w_{2}$

A) $\underline{u}_{1}>w_{2}$

First, we can show that there is an optimal relational contract with $w_{2}=\frac{\eta}{(1+\eta)} \underline{u}_{1}$. To the contrary, assume $w_{2}>\frac{\eta}{(1+\eta)} \underline{u}_{1}$. Then, (PC2) is slack, and the principal can decrease $w_{2}$ by $\varepsilon$ and increase $b_{2}$ by $\delta \varepsilon$. This operation tightens (PC2), but keeps (IC2) and (EC) unaffected and increases profits. If $w_{2}-\frac{\eta}{(1+\eta)} \underline{u}_{1}<0,(\mathrm{IC} 2)$ is slack and $b_{2}>0$. Then, the principal can increase $w_{2}$ by $\varepsilon$ and reduce $b_{2}$ by $\varepsilon$, which relaxes (EC).

If $w_{2}=\frac{\eta}{(1+\eta)} \underline{u}_{1},(\mathrm{IC} 2)$ and (PC2) are identical. They now equal $b_{2}-c\left(e_{2}\right) \geq 0$, hence it is optimal for (IC2) and (PC2) to bind because otherwise, $b_{2}$ could be reduced without violating a constraint.

Final Optimization Problem if $\underline{u}_{1}>w_{2}$

Assuming $\underline{u}_{1} \geq w_{2}$, the principal's problem is to maximize

$$
\pi=y\left(e_{1}, e_{2}\right)-c\left(e_{1}\right)-c\left(e_{2}\right)-\frac{1+2 \eta}{1+\eta} \underline{u}_{1},
$$

subject to

$$
-c\left(e_{1}\right)-c\left(e_{2}\right)+\delta\left[y\left(e_{1}, e_{2}\right)-\frac{1+2 \eta}{1+\eta} \underline{u}_{1}\right] \geq 0
$$

This immediately reveals that $e_{1}=e_{2}$ (confirming part (1) of the Proposition in case $\underline{u}_{1}>w_{2}$ ), and that the consistency requirement,

$$
m_{1} \geq m_{2} \Leftrightarrow \underline{u}_{1}+c\left(e_{1}\right) \geq \frac{\eta}{(1+\eta)} \underline{u}_{1}+c\left(e_{2}\right)
$$

is satisfied. Moreover, the left hand side of (EC) increases in $\delta$ (given the term in squared brackets 
is positive - otherwise no positive effort level can be implemented); for $\delta \rightarrow 1$, (EC) holds for $e_{1}=e_{2}=e^{F B}$. Thus, there is a $\delta^{T}(\eta) \in(0,1)$ such that $e^{F B}$ is implemented for $\delta \geq \delta^{T}(\eta)$ (confirming part (2) of the Proposition in case $\underline{u}_{1}>w_{2}$ ), and $e_{i}<e^{F B}$ for $\delta<\delta^{T}(\eta)$ (with $\delta^{T}(\eta$ ) increasing). In the latter case, effort levels $e_{1}=e_{2} \equiv e$ are characterized by the binding (EC) constraint,

$$
-2 c(e)+\delta\left[y\left(e_{1}, e_{2}\right)-\left(1+\frac{\eta}{(1+\eta)}\right) \underline{u}_{1}\right]=0
$$

with

$$
\frac{d e}{d \eta}=\frac{\delta \frac{1}{(1+\eta)^{2}} \underline{u}_{1}}{-2 c^{\prime}(e)+\delta\left[y_{1}+y_{2}\right]}<0,
$$

which is relevant for part (3) of the proposition.

For later use, note that, for $\eta \rightarrow \infty$, the (EC) constraint approaches $-2 c(e)+\delta\left[y\left(e_{1}, e_{2}\right)-2 \underline{u}_{1}\right]=0$, whereas profits approach $\pi=y\left(e_{1}, e_{2}\right)-2 c(e)-2 \underline{u}_{1}$.

Finally, since $\frac{1+2 \eta}{1+\eta}$ increases in $\eta$, profits strictly decrease in $\eta$ (which will be used in the proof to Proposition 5).

B) $\underline{u}_{1} \leq w_{2}$

Now, the set of constraints equals

$$
\begin{aligned}
\left(w_{2}+b_{2}-c\left(e_{2}\right)\right)(1+\eta)-\eta \underline{u}_{1} & \geq 0 \\
\left(w_{2}+b_{2}-c\left(e_{2}\right)\right)(1+\eta)-\eta \underline{u}_{1} & \geq(1-\delta) w_{2} \\
-c\left(e_{1}\right)-b_{2}+\delta \frac{y\left(e_{1}, e_{2}\right)-c\left(e_{1}\right)-\underline{u}_{1}-w_{2}-b_{2}}{1-\delta} & \geq 0
\end{aligned}
$$

First, we show that there exists a profit-maximizing equilibrium in which (IC2) binds. Since $w_{2} \geq 0$ (because of $w_{2} \geq \underline{u}_{1}$ ), (IC2) is tighter than (PC2). If (IC2) is slack and $w_{2}>\underline{u}_{1}$, the principal can reduce $w_{2}$ without violating a constraint. If (IC2) is slack and $w_{2}=\underline{u}_{1}$, the principal can reduce $b_{2}$; if (IC2) is slack for $w_{2}=\underline{u}_{1}$ and $b_{2}=0$, the principal can increase $e_{2}$ without violating any 
constraint (and without having to increasee agent 2's compensation). Thus, (IC2) binds, and

$$
b_{2}=c\left(e_{2}\right)+\frac{\eta \underline{u}_{1}-w_{2}(\delta+\eta)}{(1+\eta)} \geq 0
$$

Final Optimization Problem if $\underline{u}_{1} \leq w_{2}$

Assuming $\underline{u}_{1}<w_{2}$, the principal's problem is to maximize

$$
\pi=y\left(e_{1}, e_{2}\right)-c\left(e_{1}\right)-\underline{u}_{1}-c\left(e_{2}\right)-\frac{\eta \underline{u}_{1}+(1-\delta) w_{2}}{(1+\eta)}
$$

subject to

$$
-c\left(e_{1}\right)-c\left(e_{2}\right)+\delta\left(y\left(e_{1}, e_{2}\right)-\underline{u}_{1}\right)+\frac{\eta w_{2}(1-\delta)-\eta \underline{u}_{1}}{(1+\eta)},
$$

and the consistency requirements $w_{2} \in\left(\underline{u}_{1}, \frac{\underline{u}_{1}}{(1-\delta)}\right]$.

To solve this case, we set up the Lagrange function, which equals

$$
\begin{aligned}
L= & y\left(e_{1}, e_{2}\right)-c\left(e_{1}\right)-\underline{u}_{1}-c\left(e_{2}\right)-\frac{\eta \underline{u}_{1}+(1-\delta) w_{2}}{(1+\eta)} \\
& +\lambda_{E C}\left[-c\left(e_{1}\right)-c\left(e_{2}\right)+\delta\left(y\left(e_{1}, e_{2}\right)-\underline{u}_{1}\right)+\frac{\eta w_{2}(1-\delta)-\eta \underline{u}_{1}}{(1+\eta)}\right] \\
& +\lambda_{\underline{\mathrm{w} 2}}\left[w_{2}-\underline{u}_{1}\right]+\lambda_{\bar{w} 2}\left[\frac{\underline{u}_{1}}{(1-\delta)}-w_{2}\right] .
\end{aligned}
$$

There, note that the envelope condition yields

$$
\frac{d \pi}{d \eta}=\frac{\partial L}{\partial \eta}=\frac{w_{2}(1-\delta)-\underline{u}_{1}}{(1+\eta)^{2}}\left(1+\lambda_{E C}\right) \leq 0,
$$

with a strict inequality for $w_{2}(1-\delta)-\underline{u}_{1}<0$, which will be used in the proof to Proposition 5 .

First-order conditions are 


$$
\begin{aligned}
\frac{\partial L}{\partial w_{2}} & =-\frac{(1-\delta)}{(1+\eta)}+\lambda_{E C} \frac{\eta(1-\delta)}{(1+\eta)}+\lambda_{\underline{\mathrm{w} 2}}-\lambda_{\overline{w 2}}=0 \\
\frac{\partial L}{\partial e_{1}} & =y_{1}-c^{\prime}\left(e_{1}\right)+\lambda_{E C}\left[-c^{\prime}\left(e_{1}\right)+\delta y_{1}\right]=0 \\
\frac{\partial L}{\partial e_{1}} & =y_{2}-c^{\prime}\left(e_{2}\right)+\lambda_{E C}\left[-c^{\prime}\left(e_{2}\right)+\delta y_{2}\right]=0 .
\end{aligned}
$$

First, let us assume $\lambda_{\underline{\mathrm{w} 2}}=0$, which - because of the first condition - yields $\lambda_{E C}>0$. Below, we show that $w_{2}=\underline{u}_{1}$ can indeed never be optimal, which also implies that only case A) (with $w_{2}<\underline{u}_{1}$ ) can describe outcomes for a non-binding (EC) constraint, whereas case B) is only relevant for $\delta<\delta^{T}(\eta)$ (the threshold below which $e^{F B}$ cannot be implemented in case A)).

Thus,

$$
\begin{gathered}
\lambda_{E C}=\frac{\lambda_{\bar{w} 2}}{(1-\delta)} \frac{(1+\eta)}{\eta}+\frac{1}{\eta} \\
\frac{\partial L}{\partial e_{1}}=y_{1}-c^{\prime}\left(e_{1}\right)+\left(\frac{\lambda_{\bar{w} 2}}{(1-\delta)} \frac{(1+\eta)}{\eta}+\frac{1}{\eta}\right)\left[-c^{\prime}\left(e_{1}\right)+\delta y_{1}\right]=0 \\
\frac{\partial L}{\partial e_{1}}=y_{2}-c^{\prime}\left(e_{2}\right)+\left(\frac{\lambda_{\bar{w} 2}}{(1-\delta)} \frac{(1+\eta)}{\eta}+\frac{1}{\eta}\right)\left[-c^{\prime}\left(e_{2}\right)+\delta y_{2}\right]=0
\end{gathered}
$$

First, assume $\lambda_{\bar{w} 2}=0$ (i.e., $\left.w_{2}(1-\delta)-\underline{u}_{1}<0\right)$, then

$$
\begin{aligned}
& \lambda_{E C}=\frac{1}{\eta} \\
& \eta\left(y_{1}-c^{\prime}\left(e_{1}\right)\right)-c^{\prime}\left(e_{1}\right)+\delta y_{1}=0 \\
& \eta\left(y_{2}-c^{\prime}\left(e_{2}\right)\right)-c^{\prime}\left(e_{2}\right)+\delta y_{2}=0,
\end{aligned}
$$

hence

$$
e_{1}=e_{2}
$$


Moreover,

$$
w_{2}=\frac{\underline{u}_{1}}{(1-\delta)}-\frac{(1+\eta)\left[-c\left(e_{1}\right)-c\left(e_{2}\right)+\delta\left(y\left(e_{1}, e_{2}\right)-\underline{u}_{1}\right)\right]}{\eta(1-\delta)},
$$

with

$$
\frac{d e_{i}}{d \eta}=-\frac{\left(y_{i}-c^{\prime}\left(e_{i}\right)\right)}{\left(y_{i i}-c^{\prime \prime}\left(e_{i}\right)\right) \eta+\delta y_{i i}-c^{\prime \prime}\left(e_{i}\right)}>0,
$$

which is relevant for part (3) of the proposition, and

$$
\begin{aligned}
\frac{d w_{2}}{d \eta}= & \frac{\left[-c\left(e_{1}\right)-c\left(e_{2}\right)+\delta\left(y\left(e_{1}, e_{2}\right)-\underline{u}_{1}\right)\right]}{\eta^{2}(1-\delta)} \\
& -\frac{(1+\eta) 2\left[-c^{\prime}\left(e_{i}\right)+\delta y_{i}\right]}{\eta(1-\delta)} \frac{d e_{i}}{d \eta}>0
\end{aligned}
$$

which will be used in the proof to Proposition 4.

For $\eta \rightarrow \infty, e_{i}$ would approach $e^{F B}$, in which case $-c\left(e_{1}\right)-c\left(e_{2}\right)+\delta\left(y\left(e_{1}, e_{2}\right)-\underline{u}_{1}\right)$ would become negative (since $\lambda_{E C}>0$ if $\lambda_{\bar{w} 2}=0$ ). Thus, for $\underline{u}_{1}<w_{2}$ there is a $\hat{\eta}-$ defined as the value where $-c\left(e_{1}\right)-c\left(e_{2}\right)+\delta\left(y\left(e_{1}, e_{2}\right)-\underline{u}_{1}\right)=0-$ such that $w_{2}=\underline{u}_{1} /(1-\delta)$ for $\eta \geq \hat{\eta}$. Finally, for $w_{2}=\underline{u}_{1} /(1-\delta)$, profits are

$$
\pi=y\left(e_{1}, e_{2}\right)-c\left(e_{1}\right)-c\left(e_{2}\right)-2 \underline{u}_{1},
$$

and the (EC) equals

$$
-c\left(e_{1}\right)-c\left(e_{2}\right)+\delta\left[y\left(e_{1}, e_{2}\right)-\underline{u}_{1}\right]=0 .
$$

Therefore, $e_{1}=e_{2}$, confirming part (1) of the Proposition also for $\underline{u}_{1} \leq w_{2}$. Moreover, for $\eta \geq \hat{\eta}$, $d e_{i} / d \eta=d w_{2} / d \eta=0$. 


\section{Optimal Relational Contract Under Transparency}

First, we show that $w_{2}=\underline{u}_{1}$ cannot be optimal. If $w_{2}=\underline{u}_{1}$, profits are

$$
\pi=y\left(e_{1}, e_{2}\right)-c\left(e_{1}\right)-c\left(e_{2}\right)-\frac{2+2 \eta-\delta}{(1+\eta)} \underline{u}_{1},
$$

and the (EC) constraint equals

$$
-c\left(e_{1}\right)-c\left(e_{2}\right)+\delta y\left(e_{1}, e_{2}\right)-\delta \frac{1+2 \eta}{(1+\eta)} \underline{u}_{1} .
$$

In case A) with $w_{2}=\frac{\eta}{(1+\eta)} \underline{u}_{1}$, profits are

$$
\pi=y\left(e_{1}, e_{2}\right)-c\left(e_{1}\right)-c\left(e_{2}\right)-\frac{1+2 \eta}{(1+\eta)} \underline{u}_{1},
$$

and the (EC) equals

$$
-c\left(e_{1}\right)-c\left(e_{2}\right)+\delta y\left(e_{1}, e_{2}\right)-\delta \frac{1+2 \eta}{(1+\eta)} \underline{u}_{1} \geq 0 .
$$

For identical effort levels, (EC) constraints in both cases are identical, whereas profits in case A) are strictly larger. Therefore, a wage $w_{2}=\underline{u}_{1}$ is dominated by a wage $w_{2}=\frac{\eta}{(1+\eta)} \underline{u}_{1}$.

Now, we show that case B) can only be optimal if $\delta<\delta^{T}(\eta)$, i.e., if $e^{F B}$ cannot be implement in case A). Moreover, we demonstrate that, if $\delta<\delta^{T}(\eta)$, there exist values $\underline{\eta}$ and $\bar{\eta}$, with $0<\underline{\eta} \leq \bar{\eta}$, such that

- $w_{2}=\frac{\eta}{(1+\eta)} \underline{u}_{1}$ for $\eta \leq \underline{\eta}$ (case A)

- $w_{2} \in\left(\underline{u}_{1}, \underline{u}_{1} /(1-\delta)\right)$ for $\eta \in(\underline{\eta}, \bar{\eta})$ (case B)

- $w_{2}=\underline{u}_{1} /(1-\delta)$ for $\eta \geq \bar{\eta}$. (case B) 
To prove existence of $\bar{\eta}$, note that we have already shown that, in case B), setting $w_{2}=\underline{u}_{1} /(1-\delta)$ is optimal if $\eta$ is sufficiently large. With $w_{2}=\underline{u}_{1} /(1-\delta)$, profits are

$$
\pi=y\left(e_{1}, e_{2}\right)-c\left(e_{1}\right)-c\left(e_{2}\right)-2 \underline{u}_{1},
$$

and the (EC) equals

$$
-c\left(e_{1}\right)-c\left(e_{2}\right)+\delta\left[y\left(e_{1}, e_{2}\right)-\underline{u}_{1}\right]=0 .
$$

In case A) with $w_{2}=\frac{\eta}{(1+\eta)} \underline{u}_{1}$, profits are

$$
\pi=y\left(e_{1}, e_{2}\right)-c\left(e_{1}\right)-c\left(e_{2}\right)-\frac{1+2 \eta}{(1+\eta)^{2}} \underline{u}_{1}
$$

and the (EC) equals

$$
-c\left(e_{1}\right)-c\left(e_{2}\right)+\delta y\left(e_{1}, e_{2}\right)-\delta \frac{1+2 \eta}{(1+\eta)} \underline{u}_{1} \geq 0 .
$$

$\frac{1+2 \eta}{(1+\eta)}$ is strictly increasing in $\eta, \lim _{\eta \rightarrow 0} \frac{1+2 \eta}{1+\eta}=1$ and $\lim _{\eta \rightarrow \infty} \frac{1+2 \eta}{1+\eta}=2$, thus profits in case A) are larger for given levels of effort, whereas the (EC) constraint in B) allows for higher effort levels. If $\eta$ is small, the former effect dominates, whereas the latter can dominate if $\eta$ is sufficiently large, however only if $e^{F B}$ cannot be implemented in case A) because otherwise a higher effort would not increase profits. This confirms that case B) can only be optimal if $\delta<\delta^{T}(\eta)$, hence part (2) of the proposition. It also implies that, for a higher wage than $\frac{\eta}{(1+\eta)} \underline{u}_{1}$ to be optimal, effort must be discretely larger (which is displayed in Figure 1). All this establishes the existence of $\bar{\eta}$. Note that either $\bar{\eta}>\underline{\eta}$ or $\bar{\eta}=\underline{\eta}$ is possible. In any case, agent 2 receives a social comparison premium and $w_{2}+b_{2}-c\left(e_{2}\right)>0$.

Finally, part (3) of the proposition follows from the comparative statics on effort conducted above. 


\section{Proof of Proposition 4.}

In the proof to Proposition 3, we have shown that $e_{1}=e_{2}, w_{1}=\underline{u}_{1}$, and $b_{1}=c\left(e_{1}\right)$ for all levels of $\delta$ and $\eta$. Moreover, we have shown that $w_{2}=\frac{\eta}{(1+\eta)} \underline{u}_{1}$ and $b_{2}=c\left(e_{2}\right)$ in case A), which holds if $\delta \geq \delta^{T}(\eta)$ (the critical threshold above which $e^{F B}$ can be implemented) or if $\delta<\delta^{T}(\eta)$ and $\eta \leq \eta$. This confirms part (1) of the proposition. We have also shown that $d e_{i} / d \eta<0$ if $\delta<\delta^{T}(\eta)$ and $\eta \leq \eta$. Thus,

$$
\frac{d b_{i}}{d \eta}=c^{\prime}\left(e_{i}\right) \frac{d e_{i}}{d \eta} \leq 0
$$

with a strict inequality if $e_{i}<e^{F B}$, i.e., if $\delta<\delta^{T}(\eta)$.

In case B), which holds if $\delta<\delta^{T}(\eta)$ and $\eta>\underline{\eta}, w_{2}>\underline{u}_{1}$ by assumption, thus

$$
b_{2}=c\left(e_{2}\right)+\frac{\eta \underline{u}_{1}-w_{2}(\delta+\eta)}{(1+\eta)}<c\left(e_{2}\right)-\frac{\delta \underline{u}_{1}}{(1+\eta)}<c\left(e_{2}\right)
$$

Moreover, in the proof to Proposition 3 we have shown that, for $\eta \in(\underline{\eta}, \bar{\eta}), d e_{i} / d \eta>0$ and $d w_{2} / d \eta>0$. Then,

$$
\frac{d b_{1}}{d \eta}=c^{\prime}\left(e_{i}\right) \frac{d e_{i}}{d \eta}>0
$$

and

$$
\begin{aligned}
\frac{d b_{2}}{d \eta}= & \frac{\underline{u}_{1}-w_{2}(1-\delta)}{(1+\eta)^{2}} \\
& +c^{\prime}(e) \frac{d e}{d \eta}-\frac{(\delta+\eta)}{(1+\eta)} \frac{d w_{2}}{d \eta} \\
= & \delta \frac{w_{2}-\frac{\underline{u}_{1}}{(1-\delta)}}{(1+\eta) \eta}-c^{\prime}(e) \frac{d e}{d \eta}<0
\end{aligned}
$$

Finally, for $\eta \geq \bar{\eta}, d e_{i} / d \eta=0$, thus $d b_{1} / d \eta=0$. Moreover, $w_{2}=\underline{u}_{1} /(1-\delta)$ and $b_{2}=c(e)-$ $\underline{u}_{1} \delta /(1-\delta)$, thus $d b_{2} / d \eta=d w_{2} / d \eta=0$. 


\section{Proof of Proposition 5.}

For this proof, we use the superscript " $T$ " to denote effort and profits under transparency, and " $S$ " for the respective outcomes under secrecy.

Recall that we have shown (in the proof to Proposition 2) that there exist thresholds $\bar{\delta}_{i} \in(0,1)$, with $\bar{\delta}_{2}<\bar{\delta}_{1}$, such that $e_{i}=e^{F B}$ if $\delta \geq \bar{\delta}_{i}$, whereas $e_{i}<e^{F B}$ and determined by the binding (ECi) constraint if $\delta<\bar{\delta}_{i}$. Thus, for $\delta \geq \bar{\delta}_{1}$ first-best effort levels can be implemented with secrecy. In this case, $e^{F B}$ can also be implemented with transparency, however $\pi^{T}\left(e^{F B}\right)=y\left(e_{1}^{F B}, e_{2}^{F B}\right)-$ $c\left(e_{1}^{F B}\right)-c\left(e_{2}^{F B}\right)-\frac{1+2 \eta}{(1+\eta)} \underline{u}_{1}<\pi^{S}\left(e^{F B}\right)$. This confirms the first part of the proposition.

Now, assume that $\delta<\bar{\delta}_{1}$. In the proof to Proposition 3, we have shown that, under transparency, profits are decreasing in $\eta$. Thus, we first show that transparency dominates secrecy for $\eta \rightarrow 0$ and then consider larger values: In the proof to Proposition 2, we have shown that, for $\delta<\bar{\delta}_{1}$, $e_{1}^{S}<e^{F B}, e_{2}^{S} \leq e^{F B}$, and $\pi=y\left(e_{1}^{S}, e_{2}^{S}\right)-c\left(e_{1}^{S}\right)-c\left(e_{2}^{S}\right)-\underline{u}_{1}$.

Moreover, (EC) constraints under secrecy equal

$$
\begin{aligned}
-c\left(e_{1}^{S}\right)+\delta\left(y\left(e_{1}^{S}\right)-\underline{u}_{1}\right) & \geq 0 \\
-c\left(e_{2}^{S}\right)+\delta y\left(e_{2}^{S}\right) & \geq 0 .
\end{aligned}
$$

With transparency and small $\eta, \pi^{T}=y\left(e_{1}^{T}, e_{2}^{T}\right)-c\left(e_{1}^{T}\right)-c\left(e_{2}^{T}\right)-\frac{1+2 \eta}{1+\eta} \underline{u}_{1}$, and the (EC) constraint equals

$$
-c\left(e_{1}\right)-c\left(e_{2}\right)+\delta y\left(e_{1}, e_{2}\right)-\delta \frac{1+2 \eta}{(1+\eta)} \underline{u}_{1} \geq 0 .
$$

First, note that, for a given $e^{T}, \pi^{T}$ and the left hand side of (ECT) are decreasing in $\eta$. Now, we show that $\delta^{T}(\eta)<\bar{\delta}_{1}$ for $\eta \rightarrow 0$ (recall that $\delta^{T}(\eta)$ is the discount factor above which $e^{F B}$ can be implemented under transparency). At $\bar{\delta}_{1}$, (ECS2) is slack, thus (ECT) - which is the sum of (ECS1) and (ECS2) for $\eta \rightarrow 0-$ is slack as well. Thus, for $\delta^{T}(\eta) \geq \bar{\delta}_{2}$ and $\eta \rightarrow 0$, transparency 
allows us to increase $e_{1}$ without having to reduce $e_{2}$, implying that transparency is optimal in this case. Now, assume that $\delta<\bar{\delta}_{2}$, hence both (ECS) constraints bind. Moreover, $e_{2}^{S}>e_{1}^{S}$ for $\eta \rightarrow 0$. It is immediate that these levels also satisfy (ECT), hence $\pi^{T} \geq \pi^{S}$. Moreover, for $\eta \rightarrow 0$ the (uniquely) optimal implimentable effort levels under transparency are characterized by $e_{1}^{T}=e_{2}^{T}$ (see the proof to Proposition 3), thus (due to concavity of the profit function)

$$
\pi^{T}>\pi^{S}
$$

if $\delta<\bar{\delta}_{1}$ and $\eta \rightarrow 0$.

Now, we show that secrecy can be optimal for large $\eta$. Because $\pi^{T}$ is decreasing in $\eta$, it is bounded below by profits if $\eta \geq \bar{\eta}$, in which case $w_{2}^{S}=\underline{u}_{1} /(1-\delta)$ and

$$
\pi^{T}=y\left(e_{1}^{T}\right)+y\left(e_{2}^{T}\right)-2 \underline{u}_{1}-c\left(e_{1}^{T}\right)-c\left(e_{2}^{T}\right)
$$

For this case, the (EC) constraint under transparency equals

$$
-c\left(e_{1}^{T}\right)-c\left(e_{2}^{T}\right)+\delta\left(y\left(e_{1}^{T}\right)+y\left(e_{2}^{T}\right)-\underline{u}_{1}\right) \geq 0,
$$

with $e_{1}^{T}=e_{2}^{T}$. Now,

$$
\pi^{S}=y\left(e_{1}^{S}\right)+y\left(e_{2}^{S}\right)-\underline{u}_{1}-c\left(e_{1}^{S}\right)-c\left(e_{2}^{S}\right),
$$

thus transparency dominates secrecy for $\delta<\bar{\delta}_{1}$ if

$$
\begin{gathered}
\left(y\left(e_{1}^{T}\right)-c\left(e_{1}^{T}\right)\right)-\left(y\left(e_{1}^{S}\right)-c\left(e_{1}^{S}\right)\right) \\
\geq \underline{u}_{1}+\left(y\left(e_{2}^{S}\right)-c\left(e_{2}^{S}\right)\right)-\left(y\left(e_{2}^{T}\right)-c\left(e_{2}^{T}\right)\right),
\end{gathered}
$$

which might or might not hold. For example, one can show that with a quadratic cost and a linear output function, this condition is never satisfied. Then, there indeed exists a $\tilde{\eta}$ such that secrecy dominates transparency if $\eta>\tilde{\eta}$. 
To demonstrate that the condition might be satisfied and transparency dominate secrecy even for high $\eta$ (if $\delta<\bar{\delta}_{1}$ ), assume $y(e)=e$ and $c(e)=e^{k} / k$, with $k \geq 3$. Moreover, assume $\underline{u}_{1}=1 / k$ and that $\delta$ is at the level characterized by $-2 c\left(e^{F B}\right)+\delta\left(2 y\left(e^{F B}\right)-\underline{u}_{1}\right)=0$. Therefore, $e^{T}=e_{2}^{S}=e^{F B}$, whereas $e_{1}<e^{F B}$ and characterized by $-c\left(e_{1}^{S}\right)+\delta\left(y\left(e_{1}^{S}\right)-\underline{u}_{1}\right)=0$. Thus, $e^{F B}=1$, and condition (3) becomes

$$
\begin{aligned}
& \frac{2 k-5}{k} \\
\geq & \frac{2 k-3}{k} e_{1}^{S} .
\end{aligned}
$$

For $k \rightarrow \infty$, this condition becomes

$$
1 \geq e_{1}^{S}
$$

which is true.

\section{Proof of Proposition 6.}

As in the proof to proposition 4, we use the superscript " $T$ " to denote effort under transparency, and " $S$ " for effort under secrecy. In the proof to Proposition 3, we have shown that the effort under transparency is lowest at $\eta=\underline{\eta}$, i.e., if $w_{2}=[\eta /(1+\eta)] \underline{u}_{1}$ is optimal. Then, (EC) equals

$$
-2 c\left(e^{T}\right)+\delta\left[2 y\left(e^{T}\right)-\frac{1+2 \eta}{1+\eta} \underline{u}_{1}\right] \geq 0
$$

If $\delta \geq \bar{\delta}_{1},-c\left(e^{F B}\right)+\delta\left(y\left(e^{F B}\right)-\underline{u}_{1}\right) \geq 0$, and $e_{1}^{S}=e^{F B}$. Since $(1+2 \eta) /(1+\eta)<2$, the (EC) constraint under transparency also holds at first-best effort if $\delta \geq \bar{\delta}_{1}$, confirming part (1) of the proposition.

If $\delta<\bar{\delta}_{1}, e_{1}^{S}<e^{F B}$ and characterized by $-c\left(e_{1}^{S}\right)+\delta\left(y\left(e_{1}^{S}\right)-\underline{u}_{1}\right)=0 .(1+2 \eta) /(1+\eta)<2$ now implies $e^{T}>e_{1}^{S}$. Hence transparency laws increase agent 1's effort and, also his bonus (since 
$\left.b_{1}=c\left(e_{1}\right)\right)$, whereas $w_{1}\left(=\underline{u}_{1}\right)$ remains unaffected.

To show that transparency laws (weakly) reduce agent 2's effort, recall that $e_{2}^{S}$ is constrained by $-c\left(e_{2}^{S}\right)+\delta y\left(e_{2}^{S}\right) \geq 0$. Under transparency, effort is constrained by (4) if $\eta$ is low. For high $\eta$, maximum effort can be implemented with $w_{2}=\underline{u}_{1} /(1-\delta)$, in which case effort is constrained by

$$
-2 c\left(e^{T}\right)+\delta\left(2 y\left(e^{T}\right)-\underline{u}_{1}\right) \geq 0 .
$$

In both cases, implementable effort is smaller than under secrecy. Thus, if $e_{2}^{S}<e^{F B}$, agent 2's equilibrium effort under transparency is smaller than under secrecy.

The increase in output follows from the concavity of $y(\cdot)$ and the convexity of $c(\cdot)$.

The consequences for agent 2's compensation follow as well: In the proof to Proposition 2 we have shown that $w_{2}^{S}=0$ and $b_{2}^{S}=c\left(e_{2}^{S}\right)$. In the proof to Proposition 3 we have shown that $w_{2}^{T}>0$, thus transparency laws increase agent 2's salary. We have also shown that $b_{2}^{T}=c\left(e^{T}\right)$ if $\eta$ is small and that $b_{2}^{T}<c\left(e^{T}\right)$ if $\eta$ is large. Since $e^{T}<e_{2}^{S}$ if $e_{2}^{S}<e^{F B}$, agent 2's bonus then is reduced by transparency laws.

\section{Proof of Proposition 7.}

With effort always being observable, outcomes under transparency are as in the main part. Under secrecy, the optimization problem becomes to maximize

$$
\pi=y\left(e_{1}, e_{2}\right)-w_{1}-w_{2}-b_{1}-b_{2}
$$

in every period, subject to the following constraints. 


$$
\begin{aligned}
w_{1}+b_{1}-c\left(e_{1}\right)-\max \left\{0, \eta\left(c\left(e_{1}\right)-c\left(e_{2}\right)\right)\right\} & \geq \underline{u}_{1} \\
w_{2}+b_{2}-c\left(e_{2}\right)-\max \left\{0, \eta\left(c\left(e_{2}\right)-c\left(e_{1}\right)\right)\right\} & \geq 0 \\
b_{1}-c\left(e_{1}\right)-\max \left\{0, \eta\left(c\left(e_{1}\right)-c\left(e_{2}\right)\right)\right\}+\delta \frac{u_{1}}{1-\delta} & \geq \delta \frac{\underline{u}_{1}}{1-\delta} \\
b_{2}-c\left(e_{2}\right)-\max \left\{0, \eta\left(c\left(e_{2}\right)-c\left(e_{1}\right)\right)\right\}+\delta \frac{u_{2}}{1-\delta} & \geq 0 \\
-b_{1}+\delta \frac{\pi}{1-\delta} & \geq \delta \frac{\tilde{\pi}_{2}}{1-\delta} \\
-b_{2}+\delta \frac{\pi}{1-\delta} & \geq \delta \frac{\tilde{\pi}_{1}}{1-\delta} .
\end{aligned}
$$

There, $\tilde{\pi}_{2}$ maximizes $y\left(\tilde{e}_{2}\right)-c\left(\tilde{e}_{2}\right)$, subject to $-c\left(\tilde{e}_{2}\right)+\delta y\left(\tilde{e}_{2}\right) \geq 0, \tilde{\pi}_{1}$ maximizes $y\left(\tilde{e}_{1}\right)-c\left(\tilde{e}_{1}\right)-$ $\underline{u}_{1}$, subject to $-c\left(\tilde{e}_{1}\right)+\delta\left(y\left(\tilde{e}_{1}\right)-\underline{u}_{1}\right) \geq 0$.

It is immediate that $c\left(e_{2}\right) \geq c\left(e_{1}\right)$ and binding (PC1) and (IC1) constraints are optimal. Thus, agent 2's (IC) and (PC) constraints become

$$
\begin{gathered}
w_{2}+b_{2}-c\left(e_{2}\right)-\eta\left(c\left(e_{2}\right)-c\left(e_{1}\right)\right) \geq 0 \\
w_{2}+b_{2}-c\left(e_{2}\right)-\max \left\{0, \eta\left(c\left(e_{2}\right)-c\left(e_{1}\right)\right)\right\} \geq w_{2}(1-\delta) .
\end{gathered}
$$

Moreover, (IC2) and (PC2) bind (otherwise, payments to agent 2 could be reduced without violating any constraint), hence $w_{2}=0$ and $b_{2}=c\left(e_{2}\right)+\max \left\{0, \eta\left(c\left(e_{2}\right)-c\left(e_{1}\right)\right)\right\}$.

Thus, the optimization problem becomes to maximize

$$
\pi=y\left(e_{1}, e_{2}\right)-\underline{u}_{1}-c\left(e_{1}\right)-c\left(e_{2}\right)-\max \left\{0, \eta\left(c\left(e_{2}\right)-c\left(e_{1}\right)\right)\right\}
$$

in every period, subject to 


$$
\begin{aligned}
-c\left(e_{1}\right)+\delta\left[y\left(e_{1}\right)-\underline{u}_{1}-\max \left\{0, \eta\left(c\left(e_{2}\right)-c\left(e_{1}\right)\right)\right\}\right] & \geq \delta\left[y\left(\tilde{e}_{2}\right)-c\left(\tilde{e}_{2}\right)-\left(y\left(e_{2}\right)-c\left(e_{2}\right)\right)\right] \\
-c\left(e_{2}\right)-\max \left\{0, \eta\left(c\left(e_{2}\right)-c\left(e_{1}\right)\right)\right\}+\delta y\left(e_{2}\right) & \geq \delta\left[y\left(\tilde{e}_{1}\right)-c\left(\tilde{e}_{1}\right)-\left(y\left(e_{1}\right)-c\left(e_{1}\right)\right)\right]
\end{aligned}
$$

For $e_{1}=e_{2}=\tilde{e}_{1}=\tilde{e}_{2}=e^{F B}$, constraints become

$$
\begin{aligned}
-c\left(e^{F B}\right)+\delta\left[y\left(e^{F B}\right)-\underline{u}_{1}\right] & \geq 0 \\
-c\left(e^{F B}\right)+\delta y\left(e^{F B}\right) & \geq 0 .
\end{aligned}
$$

These constraints are identical to before, when effort levels are private (see the proof to Proposition 2). Thus, the relevant thresholds $\bar{\delta}_{i}$ above which $e^{F B}$ can be implemented, are the same as in Proposition 2 (and $\bar{\delta}_{2}<\bar{\delta}_{1}$ ), confirming that secrecy continues to be optimal if $\delta$ is sufficiently large.

Now assume that $\delta<\bar{\delta}_{1}$, thus $e_{1}<e^{F B}$. In the following, we show that effort and profits are lower than when effort levels are private. There, we distinguish between the two cases $e_{2}=e_{1}$ and $e_{2}>e_{1}$ (and later demonstrate that both cases can indeed be optimal).

If $e_{2}=e_{1}<e^{F B}$, profits are

$$
\pi=y\left(e_{i}\right)+y\left(e_{i}\right)-\underline{u}_{1}-2 c\left(e_{i}\right)
$$

and (EC) constraints become

$$
\begin{aligned}
-c\left(e_{i}\right)+\delta\left[y\left(e_{i}\right)-\underline{u}_{1}\right] & \geq \delta\left[y\left(\tilde{e}_{2}\right)-c\left(\tilde{e}_{2}\right)-\left(y\left(e_{i}\right)-c\left(e_{i}\right)\right)\right] \\
-c\left(e_{i}\right)+\delta y\left(e_{i}\right) & \geq \delta\left[y\left(\tilde{e}_{1}\right)-c\left(\tilde{e}_{1}\right)-\left(y\left(e_{i}\right)-c\left(e_{i}\right)\right)\right] .
\end{aligned}
$$

Because $\tilde{e}_{2}>\tilde{e}_{1}$ (which follows from $\delta<\bar{\delta}_{1}$ ) and $\underline{u}_{1}>0$, (EC1) is (ceteris paribus) tighter than (EC2) and therefore determines the effort level $e_{i}$. It follows that $e_{i}<\tilde{e}_{2}$. Therefore, for $e_{2}=e_{1}$ 
effort and profits are lower than when effort levels are private.

Now, assume that $e_{2}>e_{1}$. Then, the principal maximizes

$$
\pi=y\left(e_{1}, e_{2}\right)-\underline{u}_{1}-c\left(e_{1}\right)-c\left(e_{2}\right)-\eta\left(c\left(e_{2}\right)-c\left(e_{1}\right)\right)
$$

in every period, subject to

$$
\begin{aligned}
-c\left(e_{1}\right)+\delta\left(y\left(e_{1}\right)-\underline{u}_{1}\right) & \geq \delta\left[y\left(\tilde{e}_{2}\right)-c\left(\tilde{e}_{2}\right)-\left(y\left(e_{2}\right)-c\left(e_{2}\right)\right)+\eta\left(c\left(e_{2}\right)-c\left(e_{1}\right)\right)\right] \\
-c\left(e_{2}\right)+\delta y\left(e_{2}\right) & \geq \delta\left[y\left(\tilde{e}_{1}\right)-c\left(\tilde{e}_{1}\right)-\left(y\left(e_{1}\right)-c\left(e_{1}\right)\right)\right]+\eta\left(c\left(e_{2}\right)-c\left(e_{1}\right)\right)
\end{aligned}
$$

$e_{i} \leq \tilde{e}_{i}$ because only bilateral relational contracts are feasible. Thus, the right hand sides of both constraints are strictly positive and (EC) constraints ceteris paribus are tighter than when effort levels are private. Therefore, $e_{1}<\tilde{e}_{1}$. Moreover, $e_{2}<\tilde{e}_{2}$ if $\tilde{e}_{2}<e^{F B}$. Only if $\tilde{e}_{2}=e^{F B}$, it is potentially possible that $e_{2}=\tilde{e}_{2}=e^{F B}$, however profits are smaller in any case.

We conclude this proof by showing that both cases, $e_{2}=e_{1}$ and $e_{2}>e_{1}$, are potentially optimal if $\delta<\bar{\delta}_{1}$. To do so, we take $e_{2}=e_{1}$ as our starting point (i.e., (EC2) is slack) and explore the consequences of a marginal increase of $e_{2}, d e_{2}$. Applying the total differential to the binding (EC1) constraint delivers us the necessary change in $e_{1}$ to keep (EC1) satisfied,

$$
-c^{\prime}\left(e_{1}\right) d e_{1}+\delta\left[y_{1} d e_{1}+y_{2} d e_{2}-c^{\prime}\left(e_{2}\right) d e_{2}-\eta\left(c^{\prime}\left(e_{2}\right) d e_{2}-c^{\prime}\left(e_{1}\right) d e_{1}\right)\right]=0
$$

Thus, $\frac{d e_{1}}{d e_{2}}=\frac{\delta\left[y_{2}-c^{\prime}\left(e_{2}\right)(1+\delta \eta)\right]}{\left[(1-\delta \eta) c^{\prime}\left(e_{1}\right)-\delta y_{1}\right]}$. There, the denominator must be positive with a binding (EC1) because otherwise, a higher $e_{1}$ would relax (EC1) and increase profits. 
Then,

$$
\begin{aligned}
\frac{d \pi}{d e_{2}} & =y_{2}-(1+\eta) c^{\prime}\left(e_{2}\right)+\left(y_{1}-(1-\eta) c^{\prime}\left(e_{1}\right)\right) \frac{d e_{1}}{d e_{2}} \\
& =(1-\delta) \frac{y_{2} c^{\prime}\left(e_{1}\right)+\delta c^{\prime}\left(e_{2}\right) y_{1} \eta-c^{\prime}\left(e_{2}\right) c^{\prime}\left(e_{1}\right)\left(1+(1+\delta) \eta-\delta \eta^{2}\right)}{\left[(1-\delta \eta) c^{\prime}\left(e_{1}\right)-\delta y_{1}\right]} \\
& =(1-\delta) \frac{c^{\prime}\left(e_{i}\right)(1+\delta \eta)\left(y_{i}-c^{\prime}\left(e_{i}\right)\right)-c^{\prime}\left(e_{i}\right) c^{\prime}\left(e_{i}\right) \eta(1-\delta \eta)}{\left[(1-\delta \eta) c^{\prime}\left(e_{i}\right)-\delta y_{i}\right]} .
\end{aligned}
$$

The numerator (and consequently the whole expression) is positive if $\eta \rightarrow 0$, hence $e_{2}>e_{1}$ if $\eta$ is sufficiently small. If $\delta \rightarrow \bar{\delta}_{1}$ and $e_{i} \rightarrow e^{F B}$ (in which case $y_{i} \rightarrow c^{\prime}\left(e_{i}\right)$ ), $d \pi / d e_{2}$ becomes $(1-\delta) c^{\prime}\left(e_{i}\right) \frac{\eta(1-\delta \eta)}{\delta-(1-\delta \eta)]}$, where the numerator (and consequently the whole expression) is negative if $\eta$ is sufficiently large. The concavity of $y(\cdot)$ and the convexity of $c(e)$ imply that also a larger increase in $e_{2}$ would not increase profits, hence $e_{2}=e_{1}$ in this case. 


\section{Appendix B: Extensions}

\section{Effort Unobservable by Peer}

Here, we provide a more extensive analysis of the case where an agent's effort is unobservable by his peer under both secrecy and transparency, in which case $u_{i}=w_{i}+b_{i}-c\left(e_{i}\right)-\eta \max \left\{0, w_{j}+b_{j}-w_{i}-b_{i}\right\}$ (with $i, j \in\{1,2\}$ ) under transparency.

In the main text, we have argued that the trade-off between secrecy and transparency illustrated by Proposition 5 continues to hold. Moreover, additional tradeoffs are present, for example pay differences cause higher social comparison costs because they are not moderated by opposite differences in effort costs. Moreover, the pay of agent 2 continues to be biased towards the fixed salary, now for all levels of $\eta$. Interestingly, a similar compensation structure now can also be optimal for agent 1 , however for a different reason. If $w_{2}+b_{2}>\underline{u}_{1}$ (which is optimal if $\eta$ is large or if $\eta$ is small and his outside option is small), agent 1 also suffers from social comparisons in case he deviates and consequently is not paid the bonus. This relaxes agent 1's (IC) constraint because a lower bonus is needed to implement a certain effort level, hence $b_{1}<c\left(e_{1}\right)$ and $\left.w_{1}>\underline{u}_{1}\right)$ can be optimal. These results are summarized in the following Proposition.

Proposition B1. Assume agents cannot observe each other's effort but that multilateral enforcement is feasible. Under transparency (1) effort levels generally are not identical anymore, with agent 2's effort higher than agent 1's effort. (2) If the employment relationship is strong (high enough $\delta$ ), agent 2's effort is above, agent 1's effort below the efficient level. Then, agent 1's effort decreases and agent 2's effort increases in the strength of social comparisons. (3) If the employment relationship is weak (low $\delta$ ), agent 1's effort continues to be inefficiently low. Agent 2 's might be above or below (or at) the efficient effort level.

For all levels of $\delta$, agent 2 receives a bonus lower than the effort cost, and a salary higher than his social comparison disutility (for high $\eta$, agent 2's bonus is even zero). The same holds for agent 1, unless social comparisons are small and his outside option is large. Only then, agent 1 receives a salary equal to his outside option and a bonus equal to his effort cost. 


\section{Proof:}

As before it will be optimal to have $w_{1}+b_{1}-w_{2}-b_{2} \geq 0$ and (PC1) and (IC1) constraints binding , which yields $w_{1}=\underline{u}_{1}+\max \left\{0, \eta\left(w_{2}+b_{2}-w_{1}\right)\right\}$ and $b_{1}=c\left(e_{1}\right)-\max \left\{0, \eta\left(w_{2}+b_{2}-w_{1}\right)\right\}$.

Therefore, $w_{1}+b_{1}-w_{2}-b_{2} \geq 0$ becomes $w_{2}+b_{2}-\underline{u}_{1} \geq 0$.

Hence, the principal maximizes

$$
\pi=y\left(e_{1}, e_{2}\right)-\underline{u}_{1}-c\left(e_{1}\right)-w_{2}-b_{2}
$$

in every period, subject to the following constraints.

$$
\begin{gathered}
w_{2}+b_{2}-c\left(e_{2}\right)-\eta\left(\underline{u}_{1}+c\left(e_{1}\right)-w_{2}-b_{2}\right) \geq 0 \\
b_{2}-c\left(e_{2}\right)-\eta\left(\underline{u}_{1}+c\left(e_{1}\right)-w_{2}-b_{2}\right)+\delta \frac{u_{2}}{1-\delta} \geq-\max \left\{0, \eta\left(\underline{u}_{1}+c\left(e_{1}\right)-w_{2}\right)\right\} \\
-c\left(e_{1}\right)+(1-\delta) \max \left\{0, \eta\left(w_{2}+b_{2}-w_{1}\right)\right\}-b_{2} \\
+\delta\left(y\left(e_{1}, e_{2}\right)-\underline{u}_{1}-w_{2}\right) \geq 0
\end{gathered}
$$

In the following, we consider the cases A) $\underline{u}_{1}+c\left(e_{1}\right)-w_{2}>0$, and B) $\underline{u}_{1}+c\left(e_{1}\right)-w_{2} \leq 0$, separately.

А) $\underline{u}_{1}+c\left(e_{1}\right)-w_{2}>0$

Now, the set of constraints becomes 


$$
\begin{aligned}
w_{2}+b_{2}-c\left(e_{2}\right)-\eta\left(\underline{u}_{1}+c\left(e_{1}\right)-w_{2}-b_{2}\right) & \geq 0 \\
w_{2}+b_{2}-c\left(e_{2}\right)-\eta\left(\underline{u}_{1}+c\left(e_{1}\right)-w_{2}-b_{2}\right) & \geq(1-\delta)\left[w_{2}(1+\eta)-\eta\left(\underline{u}_{1}+c\left(e_{1}\right)\right)\right] \\
-c\left(e_{1}\right)-b_{2}+(1-\delta) \max \left\{0, \eta\left(w_{2}+b_{2}-w_{1}\right)\right\} & \\
+\delta\left(y\left(e_{1}, e_{2}\right)-\underline{u}_{1}-w_{2}\right) & \geq 0
\end{aligned}
$$

Here, we distinguish between the cases i) $w_{2}+b_{2}-\underline{u}_{1} \leq 0$, and ii) $w_{2}+b_{2}-\underline{u}_{1}>0$.

i) $w_{2}+b_{2}-\underline{u}_{1} \leq 0$

For these parameter values, $w_{2}+b_{2}-w_{1} \leq 0, w_{1}=\underline{u}_{1}$ and $b_{1}=c\left(e_{1}\right)$. We can first show that $w_{2}=\frac{\eta}{(1+\eta)}\left(\underline{u}_{1}+c\left(e_{1}\right)\right)$ is optimal. To the contrary, assume $w_{2}(1+\eta)-\eta\left(\underline{u}_{1}+c\left(e_{1}\right)\right)>0$. Then, a reduction of $w_{2}$ by $\varepsilon$ and an increase of $b_{2}$ by $\varepsilon$ does not violate a constraint. If $w_{2}(1+\eta)-$ $\eta\left(\underline{u}_{1}+c\left(e_{1}\right)\right)<0$, (IC2) is slack and a reduction of $b_{2}$ by $\varepsilon$ accompanied by an increase of $w_{2}$ by $\delta \varepsilon$ is possible.

$w_{2}=\frac{\eta}{(1+\eta)}\left(\underline{u}_{1}+c\left(e_{1}\right)\right)$ implies that (IC2) and (PC2) both become $b_{2}(1+\eta)-c\left(e_{2}\right) \geq 0$, thus hold as equalities in a profit-maximizing contract. This confirms that $w_{2}>0$ and $b_{2}=\frac{c\left(e_{2}\right)}{(1+\eta)}<c\left(e_{2}\right)$ in this case.

Thus, the optimization problem becomes to maximize

$$
\pi=y\left(e_{1} e_{2}\right)-\left(\underline{u}_{1}+c\left(e_{1}\right)\right) \frac{1+2 \eta}{1+\eta}-\frac{c\left(e_{2}\right)}{(1+\eta)},
$$

subject to the (EC) constraint

$$
-c\left(e_{1}\right)-\frac{c\left(e_{2}\right)}{(1+\eta)}+\delta\left(y\left(e_{1}, e_{2}\right)-\underline{u}_{1}-\frac{\eta}{(1+\eta)}\left(\underline{u}_{1}+c\left(e_{1}\right)\right)\right) \geq 0
$$

and the consistency requirements $w_{1}+b_{1} \geq w_{2}+b_{2} \Leftrightarrow \underline{u}_{1}+c\left(e_{1}\right) \geq c\left(e_{2}\right)$ and $w_{2}+b_{2}-\underline{u}_{1} \leq 0 \Leftrightarrow$ $\underline{u}_{1}-\eta c\left(e_{1}\right) \geq c\left(e_{2}\right)$, where the latter is tighter. 
It is immediate that $e_{2}>e_{1}$, whether (EC) binds or not. If $\delta$ is sufficiently large, (EC) is slack and $e_{2}>e^{F B}>e_{1}$.

Finally, we show that this case is only possible if $\eta$ is sufficiently small and $\underline{u}_{1}$ sufficiently large. First, for $\eta \rightarrow \infty$, the optimization problem becomes to maximize

$$
\pi=y\left(e_{1} e_{2}\right)-2\left(\underline{u}_{1}+c\left(e_{1}\right)\right),
$$

subject to

$$
-c\left(e_{1}\right)(1+\delta)+\delta\left(y\left(e_{1}, e_{2}\right)-2 \underline{u}_{1}\right) \geq 0,
$$

hence $e_{1}>0$ and the consistency requirement $\underline{u}_{1}-\eta c\left(e_{1}\right) \geq c\left(e_{2}\right)$ is violated.

Second, for $\eta \rightarrow 0$, the optimization problem becomes to maximize

$$
\pi=y\left(e_{1} e_{2}\right)-\underline{u}_{1}-c\left(e_{1}\right)-c\left(e_{2}\right),
$$

subject to

$$
-c\left(e_{1}\right)-c\left(e_{2}\right)+\delta\left(y\left(e_{1}, e_{2}\right)-\underline{u}_{1}\right) \geq 0 .
$$

The consistency requirement $\underline{u}_{1} \geq c\left(e_{2}\right)$ holds if $\underline{u}_{1}$ is sufficiently large.

ii) $w_{2}+b_{2}-\underline{u}_{1}>0$

Now, $w_{1}=\frac{\underline{u}_{1}+\eta\left(w_{2}+b_{2}\right)}{1+\eta}$ and $b_{1}=c\left(e_{1}\right)-\eta \frac{w_{2}+b_{2}-\underline{u}_{1}}{1+\eta}$, and the optimization problem becomes to maximize

$$
\pi=y\left(e_{1}, e_{2}\right)-\underline{u}_{1}-c\left(e_{1}\right)-w_{2}-b_{2},
$$

subject to 


$$
\begin{aligned}
w_{2}+b_{2}-c\left(e_{2}\right)-\eta\left(\underline{u}_{1}+c\left(e_{1}\right)-w_{2}-b_{2}\right) & \geq 0 \\
w_{2}+b_{2}-c\left(e_{2}\right)-\eta\left(\underline{u}_{1}+c\left(e_{1}\right)-w_{2}-b_{2}\right) & \geq(1-\delta)\left[w_{2}(1+\eta)-\eta\left(\underline{u}_{1}+c\left(e_{1}\right)\right)\right] \\
-c\left(e_{1}\right)-b_{2}-\delta w_{2}+(1-\delta) \eta \frac{\left(w_{2}+b_{2}\right)-\underline{u}_{1}}{1+\eta} & \\
+\delta\left(y\left(e_{1}, e_{2}\right)-\underline{u}_{1}\right) & \geq 0
\end{aligned}
$$

First, we can show that $w_{2} \geq \frac{\eta\left(\underline{u}_{1}+c\left(e_{1}\right)\right)}{(1+\eta)}$. To the contrary, assume $w_{2}<\frac{\eta\left(\underline{u}_{1}+c\left(e_{1}\right)\right)}{(1+\eta)}$, hence (IC2) is slack. Then, the principal can oncrease $w_{2}$ by $\varepsilon$ and reduce $b_{2}$ by $\varepsilon$, which relaxes the (EC) constraint and has no direct effect on profits. Thus, $w_{2} \geq \frac{\eta\left(\underline{u}_{1}+c\left(e_{1}\right)\right)}{(1+\eta)}$ and (PC2) is slack. Also note that $w_{2}=\frac{\eta\left(u_{1}+c\left(e_{1}\right)\right)}{(1+\eta)}$ if $(\mathrm{EC})$ is slack. Moreover, there is an optimal relational contract in which (IC2) binds. Otherwise, the principal can increase $w_{2}$ by $\varepsilon$ and reduce $b_{2}$ by $\varepsilon$, which relaxes (EC). A binding (IC2) constraint yields $b_{2}=\frac{c\left(e_{2}\right)+\delta \eta\left(\underline{u}_{1}+c\left(e_{1}\right)\right)}{(1+\eta)}-\delta w_{2}$. Since $w_{2} \geq \frac{\eta\left(u_{1}+c\left(e_{1}\right)\right)}{(1+\eta)}, b_{2} \leq \frac{c\left(e_{2}\right)}{(1+\eta)}$, thus $b_{2}<c\left(e_{2}\right)$ also for this case.

Moreover, since $w_{2}+b_{2}-\underline{u}_{1}>0, b_{1}=c\left(e_{1}\right)-\eta \frac{w_{2}+b_{2}-\underline{u}_{1}}{1+\eta}<c\left(e_{1}\right)$, and $w_{1}=\underline{u}_{1}+c\left(e_{1}\right)-b_{1}>\underline{u}_{1}$. Now, the final problem is to maximize

$$
\pi=y\left(e_{1}, e_{2}\right)-\underline{u}_{1}-c\left(e_{1}\right)-(1-\delta) w_{2}-\frac{c\left(e_{2}\right)+\delta \eta\left(\underline{u}_{1}+c\left(e_{1}\right)\right)}{(1+\eta)},
$$

subject to

$$
\begin{aligned}
&-c\left(e_{1}\right)-(1+\delta \eta) \frac{c\left(e_{2}\right)+\delta \eta\left(\underline{u}_{1}+c\left(e_{1}\right)\right)}{(1+\eta)^{2}}+(1-\delta) \eta \frac{(1-\delta) w_{2}-\underline{u}_{1}}{1+\eta} \\
&+\delta\left(y\left(e_{1}, e_{2}\right)-\underline{u}_{1}\right) \geq 0
\end{aligned}
$$

and the consistency requirements $w_{2} \in\left[\eta \frac{\underline{u}_{1}+c\left(e_{1}\right)}{1+\eta}, \underline{u}_{1}+c\left(e_{1}\right)\right]$.

There, we can show that $e_{2}>e_{1}$. To do so, we plug the highest feasible value of $w_{2}$ into (EC) and 
the lowest feasible value of $w_{2}$ into $\pi$ and confirm that the weight of $c\left(e_{2}\right)$ is larger than the weight of $c\left(e_{1}\right)$ in both expressions.

At $w_{2}=\eta \frac{\underline{u}_{1}+c\left(e_{1}\right)}{1+\eta}$, which is optimal if (EC) is slack, $\pi=y\left(e_{1}, e_{2}\right)-\frac{c\left(e_{2}\right)+(1+2 \eta)\left(\underline{u}_{1}+c\left(e_{1}\right)\right)}{(1+\eta)}$, hence in the unconstrained optimum $e_{2}>e_{1}$.

At $w_{2}=\underline{u}_{1}+c\left(e_{1}\right)$, the (EC) constraint equals

$$
\begin{aligned}
&-\frac{(1+\delta \eta) c\left(e_{2}\right)+c\left(e_{1}\right)[(1+\eta)(1+\delta \eta)+\delta \eta(2-\delta+\eta)]+}{(1+\eta)^{2}} \underline{u}_{1} \delta \eta(2-\delta+\eta) \\
&+\delta\left(y\left(e_{1}, e_{2}\right)-\underline{u}_{1}\right) \geq 0
\end{aligned}
$$

hence $c\left(e_{1}\right)$ ceteris paribus has a "more negative" weight in (EC) than $c\left(e_{2}\right)$.

Finally, we demonstrate that this case is only possible if $\eta$ and $\underline{u}_{1}$ are sufficiently small.

First, we demonstrate that this case violates the additional consistency requirement $w_{1}+b_{1}-$ $w_{2}-b_{2} \geq 0$ if $\eta$ is sufficiently large. $w_{2} \rightarrow \underline{u}_{1}+c\left(e_{1}\right)$ for $\eta \rightarrow \infty$, whereas (EC) approaches $-2\left(\underline{u}_{1}+c\left(e_{1}\right)\right)+y\left(e_{1}, e_{2}\right) \geq 0$ and $\pi \rightarrow y\left(e_{1}, e_{2}\right)-2\left(\underline{u}_{1}+c\left(e_{1}\right)\right)$, thus the left hand side of (EC) becomes equivalent to $\pi$. Therefore, (EC) is slack, and the optimal $e_{2}$ approaches $\infty$ for $\eta \rightarrow \infty$, which violates the consistency requirement $w_{1}+b_{1}-w_{2}-b_{2} \geq 0 \Leftrightarrow \frac{\underline{u}_{1}+c\left(e_{1}\right)-c\left(e_{2}\right)}{(1+\eta)} \geq 0$.

Second, for $\eta \rightarrow 0$, the optimization problem maximizes

$$
\pi=y\left(e_{1}, e_{2}\right)-\underline{u}_{1}-c\left(e_{1}\right)-(1-\delta) w_{2}-c\left(e_{2}\right),
$$

subject to

$$
-c\left(e_{1}\right)-c\left(e_{2}\right)+\delta\left(y\left(e_{1}, e_{2}\right)-\underline{u}_{1}\right) \geq 0
$$

and the consistency requirements $w_{2} \in\left[0, \underline{u}_{1}+c\left(e_{1}\right)\right]$. Thus, $w_{2} \rightarrow 0$, and the optimization problem is equivalent to case i). The additional consistency requirement $c\left(e_{2}\right)>\underline{u}_{1}$ holds if $\underline{u}_{1}$ is 
sufficiently small.

For further use (we show below that case $\mathrm{A}$ is optimal for high $\delta$ ), note that cases i) and ii) coincide if (EC) constraints are slack, then maximizing

$$
\pi=y\left(e_{1}, e_{2}\right)-\frac{c\left(e_{2}\right)+(1+2 \eta)\left(\underline{u}_{1}+c\left(e_{1}\right)\right)}{(1+\eta)}
$$

with

$$
\begin{aligned}
& y_{1}-\frac{(1+2 \eta) c^{\prime}\left(e_{1}\right)}{(1+\eta)}=0 \\
& y_{2}-\frac{c^{\prime}\left(e_{2}\right)}{(1+\eta)}=0
\end{aligned}
$$

and

$$
\begin{aligned}
& \frac{d e_{1}}{d \eta}=\frac{\frac{1}{(1+\eta)^{2}} c^{\prime}\left(e_{1}\right)}{y_{11}-\frac{(1+2 \eta) c^{\prime \prime}\left(e_{1}\right)}{(1+\eta)}}<0 \\
& \frac{d e_{2}}{d \eta}=-\frac{\frac{c^{\prime}\left(e_{2}\right)}{(1+\eta)^{2}}}{y_{22}-\frac{c^{\prime \prime}\left(e_{2}\right)}{(1+\eta)}}>0 .
\end{aligned}
$$

В) $\underline{u}_{1}+c\left(e_{1}\right)-w_{2} \leq 0$

First, $\underline{u}_{1}+c\left(e_{1}\right)-w_{2} \leq 0$ implies that $w_{2}+b_{2}-\underline{u}_{1}>0$, thus $w_{1}=\frac{\underline{u}_{1}+\eta\left(w_{2}+b_{2}\right)}{1+\eta}$ and $b_{1}=c\left(e_{1}\right)-$ $\eta \frac{w_{2}+b_{2}-\underline{u}_{1}}{1+\eta}$. Second, $w_{1}+b_{1}-w_{2}-b_{2} \geq 0$ becomes $\underline{u}_{1}+c\left(e_{1}\right) \geq w_{2}+b_{2}$. Both requirements can only be satisfied for $b_{2}=0$ and $w_{2}=\underline{u}_{1}+c\left(e_{1}\right)$, which further yields $w_{1}=\underline{u}_{1}+\frac{\eta c\left(e_{1}\right)}{1+\eta}>\underline{u}_{1}$ and $b_{1}=\frac{c\left(e_{1}\right)}{1+\eta}<c\left(e_{1}\right)$.

Therefore, the optimization problem maximizes

$$
\pi=y\left(e_{1}, e_{2}\right)-2\left(\underline{u}_{1}+c\left(e_{1}\right)\right)
$$


subject to

$$
\begin{aligned}
\underline{u}_{1}+c\left(e_{1}\right)-c\left(e_{2}\right) & \geq 0 \\
\underline{u}_{1}+c\left(e_{1}\right)-c\left(e_{2}\right) & \geq\left(\underline{u}_{1}+c\left(e_{1}\right)\right)(1-\delta) \\
-\frac{(1+\delta \eta)}{(1+\eta)} c\left(e_{1}\right)+\delta\left(y\left(e_{1}, e_{2}\right)-2 \underline{u}_{1}-c\left(e_{1}\right)\right) & \geq 0 .
\end{aligned}
$$

(IC2) is tighter than (PC2), thus the latter is slack. Moreover, it is optimal to let (IC2) bind. If it did not bind, $e_{2}$ could be increased without violating any constraint. Therefore,

$$
c\left(e_{2}\right)=\delta\left(\underline{u}_{1}+c\left(e_{1}\right)\right) .
$$

\section{Optimal Arrangement Under Transparency}

Now, we assess under what conditions case A) (agent 2 suffers from social comparison disutility) or case B (no social comparison disutility) is optimal.

We have already shown that cases A) i) \& ii) are not feasible for $\eta \rightarrow \infty$, thus case B) would determine the optimal arrangement for high $\eta$.

Furthermore, we can show that one of the cases A), i.e. letting agent 2 suffer from social comparison costs, is optimal if $\delta$ is high or $\eta$ is low.

First, note that for $\eta \rightarrow 0$, the optimization problem in case B) maximizes

$$
\pi=y\left(e_{1}, e_{2}\right)-\underline{u}_{1}-c\left(e_{1}\right)-\frac{c\left(e_{2}\right)}{\delta},
$$

subject to

$$
-c\left(e_{1}\right)-c\left(e_{2}\right)+\delta\left(y\left(e_{1}, e_{2}\right)-\underline{u}_{1}\right) \geq 0
$$

In case A), the optimization problem for $\eta \rightarrow 0$ (recall that we have shown above that i) and ii) 
are equivalent in that case) maximizes

$$
\pi=y\left(e_{1} e_{2}\right)-\underline{u}_{1}-c\left(e_{1}\right)-c\left(e_{2}\right),
$$

subject to

$$
-c\left(e_{1}\right)-c\left(e_{2}\right)+\delta\left(y\left(e_{1}, e_{2}\right)-\underline{u}_{1}\right) \geq 0 .
$$

Thus, (EC) constraints are equivalent in both cases, whereas $\pi$ ceteris paribus is higher in case A), which therefore dominates for low $\eta$.

For $\delta \rightarrow 1$, case B) becomes

$$
\pi=y\left(e_{1}, e_{2}\right)-2\left(\underline{u}_{1}+c\left(e_{1}\right)\right)
$$

subject to

$$
y\left(e_{1}, e_{2}\right)-2\left(\underline{u}_{1}+c\left(e_{1}\right)\right) \geq 0 .
$$

Cases A) i) $\&$ ii) are again equivalent for $\delta \rightarrow 1$ and maximize

$$
\pi=y\left(e_{1}, e_{2}\right)-\left(\underline{u}_{1}+c\left(e_{1}\right)\right) \frac{1+2 \eta}{1+\eta}-\frac{c\left(e_{2}\right)}{(1+\eta)},
$$

subject to

$$
y\left(e_{1}, e_{2}\right)-\left(\underline{u}_{1}+c\left(e_{1}\right)\right) \frac{1+2 \eta}{1+\eta}-\frac{c\left(e_{2}\right)}{(1+\eta)} \geq 0 .
$$

To better compare both cases, we take into account that $c\left(e_{2}\right)=\underline{u}_{1}+c\left(e_{1}\right)$ in case B). Imposing this additional constraint on case A) delivers a lower bound for profits, which then become $\pi=$ $y\left(e_{1}, e_{2}\right)-2\left(\underline{u}_{1}+c\left(e_{1}\right)\right) \frac{\eta}{1+\eta}$. For given effort levels, those are larger than in case $\left.\mathrm{B}\right) ;$ therefore case A) is optimal if $\delta$ is large. 
Finally, note that transparency dominates secrecy if $\eta$ is small (and first-best effort cannot be implemented under secrecy) also with unobservable effort. With small $\eta$, social comparison costs become negligible (irrespective of whether effort is observable to peers or not), whereas the benefits of multilateral enforcement allow for higher productivity.

\section{Symmetric Social Comparisons}

Here, we briefly confirm that the principal can benefit from downward social comparisons. Under transparency, also the agent with higher material payoff experiences a disutility. Thus, the benefits of equalizing material payoffs are even larger than with asymmetric social comparisons. As before, if the principal wants to eliminate social comparison costs, she sets $w_{2}>w_{1}=\underline{u}_{1}$ and $b_{2}<b_{1}=$ $c\left(e_{1}\right)$. Performing similar operations as in the main part, the optimization problem with equal material payoffs would be to maximize

$$
\pi=y\left(e_{1}, e_{2}\right)-c\left(e_{1}\right)-c\left(e_{2}\right)-2 \underline{u}_{1},
$$

subject to

$$
-c\left(e_{1}\right)-c\left(e_{2}\right)+\delta\left(y\left(e_{1}, e_{2}\right)-\underline{u}_{1}\right)+\frac{\alpha \delta}{(1-\alpha)} \underline{u}_{1} \geq 0 .
$$

It is immediate that a higher $\alpha$ relaxes (EC) without adversely affecting profits. 